\title{
ADVANCES IN MATHEMATICAL PROGRAMMING MODELS FOR ENTERPRISE-WIDE OPTIMIZATION
}

\author{
Ignacio Grossmann \\ Center for Advanced Process Decision-making \\ Department of Chemical Engineering \\ Carnegie Mellon University \\ Pittsburgh, PA 15213
}

\begin{abstract}
Enterprise-wide optimization (EWO) is an area that lies at the interface of chemical engineering and operations research, and has become a major goal in the process industries due to the increasing pressures for remaining competitive in the global marketplace. EWO involves optimizing the operations of supply, manufacturing and distribution activities of a company to reduce costs, inventories and environmental impact, and to maximize profits and responsiveness. A major focus in EWO is the optimal operation of manufacturing facilities that often require the use of nonlinear process models. Major operational items include planning, scheduling, real-time optimization and control. We provide an overview of EWO in terms of a mathematical programming framework. We first provide a brief overview of mathematical programming techniques (mixed-integer linear and nonlinear optimization methods), as well as decomposition methods, stochastic programming and modeling systems. We then address some of the major issues involved in the modeling and solution of these problems. Finally, based on the EWO program at the Center of Advanced Process Decision-making at Carnegie Mellon, we describe several applications to show the potential of this area.
\end{abstract}

Keywords

\section{Introduction}

The chemical industry is a major component of the US economy, converting raw materials such as oil, natural gas, air, water, metals and minerals into more than 70,000 different products According to the American Chemical Council (ACC: 'Industry Fact Sheet'), over 96 percent of all manufactured goods are dependent in some way on the chemical industry. The US chemical industry produces 19 percent of the world's chemical output, amounting to US\$689 billion (The Outlook for the US Chemical Industry, KPMG, 2010). In addition, the US chemical industry is responsible for 10 percent of US merchandise exports, totaling US\$145 billion annually. The industry directly employs over 800,000 people nationwide.

Due to the increasing pressure for reducing costs, inventories and ecological footprint, and in order to remain competitive in the global marketplace, Enterprise-wide Optimization (EWO) has become a major goal of the chemical industry. The last two conferences on Foundations of Computer-Aided Process Operations in Coral Springs in January 2003, and in Boston in July, 2008, had a number of papers from a variety of process industries such as petroleum, chemical, pharmaceutical, consumer products, that referred to the goal of EWO throughout the optimization of supply chain of these industries; see http://www.cheme.cmu.edu/focapo and special issues of Computers \& Chemical Engineering, 28 (6-7) (2004) and 33 (12) (2009). The need for EWO in the process industry has also been reinforced with the announcement of the new initiative on "Advanced Manufacturing Plan" announced by President Obama (http://www.whitehouse.gov/sites/default/files/microsites/os tp/advanced-manu-fs.pdf). EWO has also been an important element of the "Smart Manufacturing" initiative led by Jim Davis and Tom Edgar https://smart-processmanufacturing.ucla.edu/

This paper is a follow-up to an earlier paper by the author (Grossmann, 2005) that outlined the major research 
challenges involved in the area of EWO for process industries. Subsequently, Varma et al, (2007) provided an alternative overview of challenges and opportunities in this area with major emphasis in the pharmaceutical industry. The present paper emphasizes the scope and application of mathematical programming techniques to EWO problems. The paper is organized as follows. We first present a general definition of Enterprise-wide Optimization, and describe an initiative that we have had in this area at the Center of Advanced Process Decision-making (CAPD) at Carnegie Mellon for the last five years. Next, we present a review of mixedinteger optimization, stochastic programming techniques, and decomposition techniques, which constitute the core of EWO models. Next, we discuss some of the main research issues that we have identified as a result of this effort. We then present several examples to illustrate the potential of new developments in EWO. Finally, we close by outlining some of the major remaining challenges in this area.

\section{Definition of Enterprise-wide Optimization}
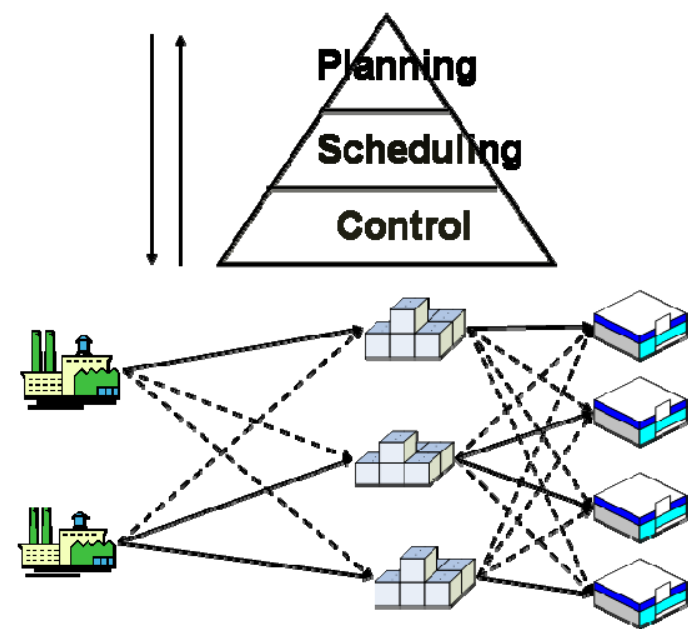

Figure 1. Major elements of Enterprise-wide Optimization

Enterprise-wide Optimization is concerned with the coordinated optimization of the operations of a supply chain (Shapiro, 2001), namely R\&D, supply of material, manufacturing and distribution of products. Process supply chains range from the ones in the petroleum industry (Shah et al., 2011) to the ones in the pharmaceutical industry (Shah, 2004), and include manufacturing as a major component (Pistikopoulos et al, 2007; Wassick, 2009). The main objectives in EWO include maximization of profits, responsiveness to customers and asset utilization, and minimization of costs, inventory levels and ecological footprint. Major operational activities include planning, scheduling, real-time optimization and control (see Fig. 1). One of the key features in EWO is integration of the information and decision-making among the various functions that comprise the supply chain of the company. Integration of information is being achieved with modern IT tools (e.g. SAP and Oracle) that allow the sharing and instantaneous flow of information along the various organizations in a company. While IT tools allow many groups in an enterprise to access the same information, these tools do not provide comprehensive decision making capabilities for optimization that account for complex trade-offs and interactions across the various functions, subsystems and levels of decision making. This means that companies are faced with the problem of deciding whether to develop their own in-house tools for integration, or make use of commercial software from vendors.

In order to realize the full potential of transactional IT tools, the development of sophisticated decision-support tools based on mathematical programming (analytical IT tools) is needed to operate the supply chain in order to yield overall optimum economic performance, as well as high levels of customer satisfaction. A major challenge that is involved in EWO of process industries is the integrated and coordinated decision-making across the various functions in a company (purchasing, manufacturing, distribution, sales), across various geographically distributed organizations (vendors, facilities and markets), and across various levels of decision-making (strategic, tactical and operational) as seen in Figure 2 (Shapiro, 2001).

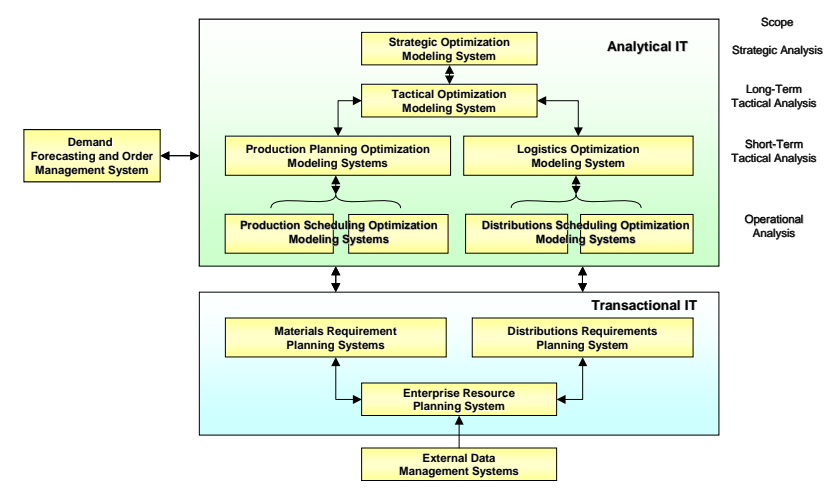

Figure 2. Transactional and Analytical IT (Tayur et al., 1999)

\section{The EWO Initiative at Carnegie Mellon}

Over the last 5 years we have organized at the Center of Advanced Process Decision-making (CAPD) at Carnegie Mellon a special interest group on Enterprise-wide Optimization. This research effort has been multidisciplinary in nature involving faculty from chemical engineering (Larry Biegler and Ignacio Grossmann at CMU), industrial engineering (Jeff Linderoth and Larry Snyder at Lehigh, and Andrew Schaefer from UPitt), and operations research (John Hooker, Nic Secomandi, Tepper Business School at CMU). The EWO group has over the years involved the following 14 companies: ABB, Air Liquide, Air Products, BP, Braskem, Cognizant, Dow Chemical, Ecopetrol, 
ExxonMobil, NOVA Chemicals, PPG, Praxair, Total, and supply chains involving logistics and distribution, Unilever.

The major goal of the EWO group at Carnegie Mellon has been to develop mathematical programming models and solution algorithms for a variety of applications, which have recently included the following:

- Batch Scheduling with Electric Power Constraints $(A B B)$

- Design of Resilient Supply Chain Networks for

Chemicals and Gases (Air Products)

- Production-Distribution Coordination for Planning of an Industrial Gases Supply Chain (Air Liquide)

- Nonlinear models for Refinery Optimization $(B P)$

- Multiperiod Scheduling of Polyproylene Production

(Braskem)

- Optimization of Gas Pipelines (Cognizant)

- Design of Reliable Integrated Sites (Dow)

- Simultaneous Scheduling and Dynamic Optimization of Batch Processes (Dow)

- Nonlinear Programming for Refinery Optimization

(Ecopetrol)

- Global Optimization of Bilinear GDP Models (ExxonMobil)

- Multistage Stochastic Programming for Design and

Planning of Oil and Gasfields (ExxonMobil)

- Chemicals: Polymerization Optimization (NOVA Chemicals

- Planning and Scheduling for Glass Production $(P P G)$

- Capacity Planning of Power Intensive Networks with

Changing Electricity Price (Praxair)

- Scheduling of Crude Oil Operations (Total)

- Scheduling of Fast Moving Consumer Goods (Unilever)

Knowledge that has emerged from these projects, which have largely emphasized planning and scheduling of a number of process systems, has been shared among the companies, and is in fact available in http://egon.cheme.cmu.edu/ewocp/slides_meetings.html.

From a mathematical point of view most of the models that have been developed correspond to mixed-integer optimization problems. However, some of the projects also involve nonlinear programming and dynamic optimization problems as well. A series of seminars has been organized around these topics and can be found in: http://egon.cheme.cmu.edu/ewocp/slides_seminars.htm.

Below we first briefly review the major mathematical programming tools that have been used in these projects. Next, we discuss some of the major challenges and issues that we have had to face in the application of these techniques in EWO projects.

\section{MILP}

A large number of optimization problems in EWO can be described by Mixed-Integer Linear Programming (MILP) models. Examples include the optimization of production operations including planning and scheduling (Pinedo, 2001; Mendez et al., 2006; Floudas and Lin, 2004; Pochet and Wolsey, 2006), optimization multiple period optimization (Grossmann, 2005). In the process industries, real world problems usually lead to largescale models, due to the size of the system under study, but also because of models that involve multiple periods. Furthermore, often new variables and equations are introduced to replace nonlinearities by piecewise linear approximations, or by performing exact linearizations (eg. product binary and continuous variables).

MILP problems may be represented by the following formulation in terms of continuous and $0-1$ variables:

$$
\begin{array}{ll}
\min & Z=c^{T} y+d^{T} x \\
\text { s.t. } & A y+B x \leq b \quad \text { (MILP) } \\
& y \in\{0,1\}, x \geq 0
\end{array}
$$

where $c, d, b$ are vectors of coefficients, and $A$ and $B$ are matrices of coefficients.

Generally MILP problems can be solved using Linear Programming (LP)-based Branch \& Bound (B\&B) solvers (Wolsey, 1998) that provide rigorous lower and upper bounds on the solution, which in turn provide information regarding the optimality of the solution. Broadly speaking the LP-based branch and bond algorithm starts with the solution of the initial relaxed LP problem at the root node in which the integer variables are treated as continuous variables. The solution to this problem provides a lower bound. If the continuous relaxation does not yields integer values for the $0-1$ variables a tree search is performed by successively enforcing integrality on the variables in the tree search and solving the corresponding LP subproblem. When a feasible solution is found this yields an upper bound. This procedure is repeated for each sub-problem, until the upper bound defined by integer solutions is equal to the lower bound given by the LP subproblems. During the search the upper and lower bounds are used to prune branches of the tree (for a detailed description see Wolsey (1998)). B\&B algorithms, however, may not be able to effectively solve large problems due to the exponential number of subproblems that may have to be solved, particularly when the LP relaxation is poor. MILP solvers have implemented more sophisticated versions denoted by Branch \& Cut (B\&C) algorithms. In these algorithms, valid inequalities denoted by cutting planes are added to the linear relaxations in order to reduce the size of the feasible space without eliminating any feasible integer solution.

In the last 10 years great progress has been made in algorithms and hardware, which has resulted in an impressive improvement of our ability to solve mixedinteger programming problems (MILPs) (Johnson et al., 2000; Bixby, 2002; Bixby, Rothberg, 2007) through codes such as CPLEX, XPRESS and GUROBI. Capitalizing on theory developed during the last 20 years, it is now possible, using off-the-shelf LP-based branch-and-bound commercial software, to solve in a few seconds MILP instances that were unsolvable 10 years ago. As an example, consider the 
classical Kondili State-Task Network MILP problem (Kondili et al.1993) with 72 binary variables, 179 continuous variables and 250 constraints. In 1987 using Kondili's B\&B with MINOS for the LP solver, it took 908 seconds and 1466 nodes in a VAX-8600 to solve this problem, while Shah et al. (1993) with a tighter constraint in place of a big-M constraint took 119 seconds and 419 nodes using a SUN Sparc. Today in 2011, using CPLEX 12.1 with a laptop Lenovo t60p only 0.14 seconds and 14 nodes were required. A more standard benchmark for MILP problems, the MIPLIB 2003 (Achterberg et al, 2006), shows that in the beginning of 2003 there were more than 30 problems with unknown optimal solution, while in the beginning of 2011 only four problems remain to be solved (http://miplib.zib.de) The current computational performance of MILP software is the result of a combination of improvements in LP solvers, preprocessing techniques for LP and MILP, linear algebra for sparse systems, cutting planes, heuristics, parallelization and faster computers (Bixby, Rothberg, 2007).

\section{MINLP Methods}

While MINLP models are still less common than MILP problems in EWO applications, there are a number of important applications that require the handling of nonlinearities, which in turn give rise to MINLP models. The most basic form of an MINLP problem is as follows (Grossmann, 2002):

$$
\begin{array}{cc}
\min Z=f(x, y) \\
\text { s.t. } \quad g_{j}(x, y) \leq 0 \quad j \in J \\
x \in X, y \in Y
\end{array}
$$

(MINLP)

where $f: R^{n} \rightarrow R^{l}, g: R^{n} \rightarrow R^{m}$ are differentiable functions, $J$ is the index set of constraints, and $x$ and $y$ are the continuous and discrete variables, respectively. In the general case the MINLP problem will also involve nonlinear equations, which we omit here for convenience in the presentation. The set $X$ commonly corresponds to a convex compact set, e.g. $X=\left\{x \mid x \in \boldsymbol{R}^{n}, D x \leq d, x^{L} \leq x \leq x^{U}\right\} ; \quad$ the discrete set $Y$ corresponds to a polyhedral set of integer points, $Y=\left\{y \mid y \in \mathbf{Z}^{m}, A y \leq a\right\}$ which in most applications is restricted to $0-1$ values, $y \in\{0,1\}^{m}$. In most applications the objective and constraint functions $f(\cdot), g(\cdot)$ are linear in $y($ e.g. fixed costs and mixed-logic constraints):

$f(x, y)=c^{T} y+r(x), g(x, y)=B y+h(x) . \quad$ The derivation of the most methods for MINLP assumes that the functions $f$ and $g$ are convex.
Methods that have addressed the solution of problem (MINLP) include first branch and bound methods (BB), which are generally a direct extension of the LP-based branch and bound method for MILP, except that in this case an NLP solver (e.g. reduced gradient, successive quadratic programming, or interior point method) is used at each node (e.g. Leyffer, 2001), while the use of cuts at this point is still rather limited (Stubbs and Mehrotra, 1999). A second class of methods includes decomposition algorithms such as Generalized Benders Decomposition (GBD) (Geoffrion, 1972) and Outer-Approximation (OA) (Duran and Grossmann, 1986; Fletcher and Leyffer, 1994), in which there is an iterative sequence of NLP subproblems with fixed 0-1 variables that yield upper bounds, and MILP master problems that yield lower bounds. Convergence in this case is achieved when bounds lie within a specified tolerance. In order to avoid the repeated sequence of MILP master problems, branch and cut methods (or LP/NLP based branch and bound) have been proposed in which a single branch and bound tree is enumerated by updating the linear approximations with solution of NLP subproblems at selected nodes (Quesada and Grossmann, 1992; Bonami et al, 2008). Finally, a variant of the decomposition methods is the Extended Cutting Plane Method (ECP) (Westerlund and Pettersson, 1995, and Westerlund and Pörn (2002)) in which the NLP subproblem is replaced by function evaluations, with which the algorithm effectively reduces to a successive MILP method.

The number of computer codes for solving MINLP problems has greatly increased in the last decade. The program DICOPT (Viswanathan and Grossmann, 1990) is an MINLP solver that is based on the outer-approximation algorithm with some extensions, and is available in the modeling system GAMS (Brooke et al., 1998). This code also uses the relaxed NLP to generate the first linearization of the first MILP master problem, with which the user need not specify an initial integer value. Also, since the lower bounding properties of the master problem cannot be guaranteed, the search for nonconvex problems is terminated when there is no further improvement in the feasible NLP subproblems. This is a heuristic that works reasonably well in many problems. A similar code to DICOPT, AAOA, is available in AIMMS. Codes that implement the branch-andbound method solving NLP subproblems at each node include the code MINLP_BB that is based on an SQP algorithm (Leyffer, 2001) and is available in AMPL, and the code SBB, which is available in GAMS (Brooke et al, 1998). The code $\alpha-$ ECP that is available in GAMS implements the extended cutting plane method by Westerlund and Pettersson (1995), including the extension by Westerlund and Pörn (2002). The code MINOPT (Schweiger and Floudas, 1998) also implements the OA and GBD methods, and applies them to mixed-integer dynamic optimization problems. The open source code Bonmin (Bonami et al, 2008) implements the branch and bound method, the outer-approximation and an extension of the LP/NLP based branch and bound method in one single framework. FilMINT (Abhishek, Linderoth and 
Leyffer, 2010) also implements a variant of the LP/NLP based branch and bound method. Codes for the global optimization that implement the spatial branch and bound method include BARON (Sahinidis, 1996), LINDOGlobal (Lindo Systems, 2010), and Couenne (Belotti et al, 2009). For a detailed treatment of global optimization problems see Tawarlamani and Sahinidis (2002)

It should be noted that solving MINLP problems is still often a non-trivial task, especially when compared to solving MILP problems. A major reason for this is the need to solve NLP subproblems (see Biegler (2010) for a review), which often require good initial points to ensure convergence. Therefore, often an approach that is used in scheduling is to reformulate the problem as an MILP by for instance using exact linearizations of products of binary and continuous variables, or using piecewise linear approximations. This, however, can only be done in relatively few cases.

\section{Logic-based Optimization Methodologies}

Disjunctive Programming. A linear programming problem that includes as special constraints disjunctions (Balas, 1979) is denoted as a disjunctive programming problem. Disjunction refer to constraints where only one set of inequalities must be satisfied but not all. These disjunctions have been used extensively in scheduling to represent for instance whether a product i precedes product $\mathrm{j}$ or vice-versa through inequalities, where each of these is expressed in terms of start times for jobs $i$ and $\mathrm{j}$ (Adams et al., 1988). Special purpose algorithms have been developed for solving disjunctive programs, the most common one being the disjunctive branch and bound method (Beaumont, 1991). Generalized disjunctive programming (GDP) corresponds to a generalization of linear or nonlinear programming model that includes disjunctions given in terms of constraints and Boolean variables that are in turn constrained through logic propositions (Raman and Grossmann, 1994). One way to solve a GDP problem is to reformulate it as a mixed-integer program, using either a big-M reformulation or a hull relaxation formulation that is given in terms of disaggregated variables with which the convex hull can be obtained for each disjunction (Lee and Grossmann, 2000). While the first produces a smaller mixed-integer program, the latter generally yields stronger lower bounds for its relaxation. Also, as has been shown by Sawaya and Grossmann (2011) and Ruiz and Grossmann (2011), the hull relaxation can be further strengthened through the application of basic steps that involve intersecting pairs of disjunctions. The advantage of starting with a GDP mode, is that it can be used as basis to derive mixedinteger programs that exhibit good relaxation properties, which is especially useful in many large-scale scheduling models. Software for GDP problems is available in the GAMS based codes LOGMIP (Vecchietti and Grosmann, 1999) and EMP (Ferris et al, 2009).

Constraint Programming. In terms of logic-based optimization methods, constraint programming offers an alternative approach for tackling scheduling problems, particularly resource-constrained scheduling problems, which occur frequently in supply-chain problems. Constraint programming $(\mathrm{CP})$ offers certain advantages in the modeling and solution of scheduling problems. The models tend to be more concise and easier to interpret since logical and combinatorial conditions are much more naturally expressed in a CP than in an MILP model (e.g., Hooker, 2000; Hooker, 2002; Milano 2003). The solvers take advantage of logical inference (constraint propagation) methods that are well suited to the combinatorial constraints that characterize scheduling problems. For instance, a CP model readily formulates sequencing problems and offers specialized propagation algorithms that exploit their structure. The integration of CP and MILP methods has shown to be effective in specialized scheduling problems. Integration allows one to attack problems in which some of the constraints are better suited to an MILP-like approach (assignment decisions because they have good continuous relaxations) and others are better suited for a $\mathrm{CP}$ approach (sequencing decisions because they "propagate well"). Hybrid methods have shown in some problems outstanding synergies that lead to order magnitude reductions in computation (Jain and Grossmann, 2001; Maravelias and Grossmann, 2004; Hooker 2007). Software for CP includes OPL (Van Hentenryck, 1999) now distributed by IBM, and CHIP, distributed by Cosytech.

\section{Stochastic Programming}

Planning under uncertainty is an important problem that arises in EWO problems. Modeling and solution of this class of problems by stochastic programming directly takes uncertainty into account in terms of probability distribution functions (Birge \& Louveaux, 1997; Sahinidis, 2004). A Stochastic Program is a mathematical program in which some of the parameters defining a problem instance are random (e.g. demand, yield). The basic idea behind stochastic programming is to make some decisions now, stage 1 , and to take some corrective action (recourse) in the future, stage 2, after revelation of the uncertainty. If there are only two stages then the problem corresponds to a 2-stage stochastic program, while in a multistage stochastic program the uncertainty is revealed sequentially, i.e. in multiple stages (periods), and the decision-maker can take corrective action over a sequence of stages. In the two-stage and multistage case the cost of the decisions and the expected cost of the recourse actions are optimized.

The uncertain parameters are commonly assumed to follow discrete probability distributions and a planning horizon consisting of a fixed number of decision points. Using these two assumptions, the stochastic process can be 
represented with scenario trees. In a scenario tree (Fig. 3-a) each node represents a possible state of the system at a given time period. Each arc represents the possible transition from one state in time period $t$ to another state in time period $t+1$, where each state is associated with the probabilistic outcome of a given uncertain parameter. A path from the root node to a leaf node represents a scenario. An alternative representation of the scenario tree was proposed by Ruszczynski (1997) where each scenario is represented by a set of unique nodes (Fig. 3-b). The horizontal lines connecting nodes in time period $t$ mean that nodes are identical as they have the same information and those scenarios are said to be indistinguishable in that time period. These horizontal lines correspond to the non-anticipativity constraints in the model that link different scenarios and prevent the problem from being decomposable.

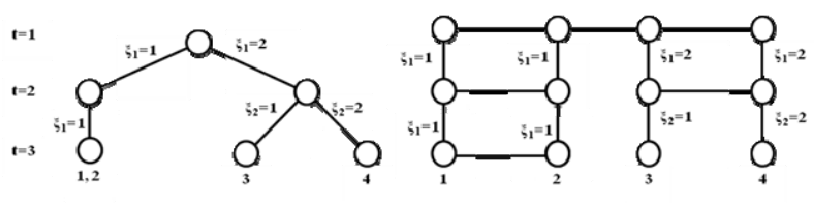

Figure 3a. Standard Scenario Tree with uncertain parameters $\xi_{1}$ and $\xi_{2}$ Figure 3-b: Alternative Scenario Tree

Jonsbraten et al. (1998) classified uncertainty in planning problems into two broad categories: exogenous uncertainty (e.g. demands) where stochastic processes are independent of planning and investment decisions and endogenous uncertainty (e.g. yields) where stochastic processes are affected by these decisions. While exogenous parameters are quite common, as for instance is the case of uncertain demands, a special type of multistage stochastic programming (MSSP) problems involve endogenous uncertainty where the structure of scenario tree is decision-dependent. As for solution methods, the simplest is to convert the problem into its deterministic equivalent form. Since this may give rise to very large-scale problems some type of decomposition method is used to tackle these problems (Birge \& Louveaux, 1997; Sahinidis, 2004).

In contrast to stochastic programming, robust optimization (Ben-Tal et al., 2009) focuses on a computationally tractable description of an uncertainty set, against which the solution is "robustified". Soyster's worst-case approach (1973) is considered to be too conservative. Bertsimas and Sim (2003) provide a computationally tractable linear formulation that allows controlling the degree of conservatism. Bertsimas and Sim's (2003) approach assumes that each uncertain parameter will be either at its nominal or worst-case value and that the total number of parameters that take their worst-case values is restricted. The main advantage of robust optimization methods is that they are much more tractable than their stochastic programming counterpart. The disadvantage is that they do not include recourse actions limiting their scope for short-term problems in which recourse is not an issue.

\section{Decomposition Techniques}

Given the large size of many EWO problems, the application of decomposition techniques is often required. Four common approaches in EWO problems are Lagrangean decomposition, Benders decomposition, bi-level decomposition and rolling horizon.

Lagrangean Decomposition (Geoffrion, 1972) is perhaps the most common technique used for decomposing problems so as to make them tractable. An excellent review on Lagrangean Relaxation and Lagrangean Decomposition can be found in Guignard (2003). Lagrangean relaxation is a useful technique for problems with complicating constraints. The basic idea consists of dualizing the complicating constraints by transferring them into the objective function and multiplying them by the Lagrange multipliers. The solution to this relaxed problem for fixed multipliers provides a lower bound for the minimization case. Furthermore, by fixing these multipliers the relaxation decomposes into subproblems (e.g. by time periods or by sites in supply chain problems; see Terrazas-Moreno et al, 2011). Using heuristics an upper bound is obtained, after which the multipliers are typically updated with a subgradient optimization method so as to maximize the dual problem. The iterations proceed until the bounds lie within a specified tolerance or a maximum number of iterations is exceeded. It should be noted that non-convexities such as binary variables in an MILP problem give rise to a duality gap, and hence termination is by exceeding a specified limit of number of iterations.

There is rich literature on the application of Benders decomposition (BD) (Benders, 1962) to optimization under uncertainty. Most of these applications model uncertainty using a stochastic programming (SP) representation (Birge and Louveaux, 1997), and apply variations of the BD algorithm. The standard decomposition technique is referred to as L-shaped decomposition (Van Slyke and Wets, 1969) in the stochastic programming literature. Straub and Grossmann (1993) proposed a nonlinear programming (NLP) model for maximizing the feasible operating region of a network with uncertain process parameters and used Generalized Benders Decomposition (GBD) (Geoffrion, 1972) to solve this problem. A similar approach was proposed by Pistikopoulos (1995) and applied by Ierapetritou et al. (1996) as a general algorithmic technique for solving a class of problems defined as process design and operations under uncertainty.

The bi-level decomposition algorithm is a specialized tailored decomposition technique. This technique was first proposed by Iyer and Grossmann (1998) for solving a multiperiod capacity expansion planning model for process networks. In the context of their problem the algorithm 
involves a master problem in the reduced variable space of binary variables to determine the selection of processes and an upper bound to the NPV. A detailed planning model is then solved for the selected processes to determine the expansion plan and a lower bound to the objective function. Special cuts are added to the master problem to accelerate the convergence. Similar bi-level decomposition schemes have been developed by Bok et al. (2000) for supply chain optimization problem, and by Erdirik-Dogan and Grossmann (2006) for the simultaneous planning and scheduling of single-stage continuous multiproduct plants.

\section{Problem 1}

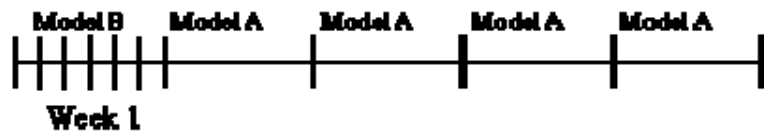

Problem 2

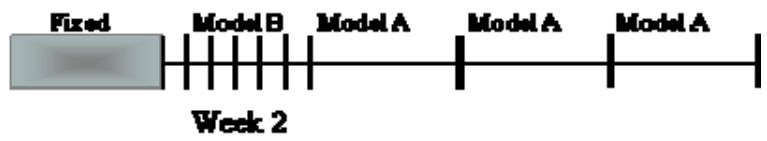

Problem 3

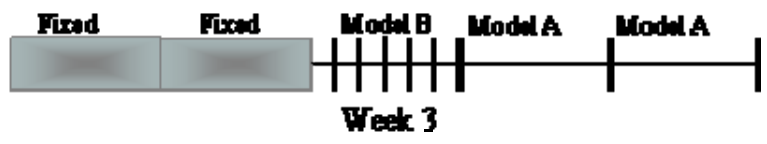

Figure 4. Application of rolling horizon with detailed model $A$ and aggregated model $B$.

Finally, another major decomposition approach relies on a rolling horizon strategy in which multiperiod problems are solved by recursively applying a more detailed model in the first time period and a simpler aggregate problem in the remaining time periods (Bassett et al, 1996; Dimitriadis et al., 1997). After each stage, the decisions in the first time period are fixed and the horizon time is effectively shrunk as seen in Fig. 4.

\section{Multiobjective Optimization}

As indicated above, EWO problems involve multiple objectives, which are often conflicting in nature (e.g. NPV vs. responsiveness, NPV vs. environmental impact), thus giving rise to trade-off or Pareto-optimal solutions. There are three main types of multiobjective optimization approaches: (1) those based in the transformation of the problem into a single-objective one (see Ehrgott, 2000), (2) the Non-Pareto approaches, which use search operators based in the objectives to be optimized and (3) Pareto approaches, which directly apply the concept of dominance (see Deb, 2008). Whereas the first approach can be easily applied in conjunction with standard exact algorithms (i.e., branch and bound), the second and third ones are better suited to work with meta-heuristics. Note that any of the traditional exact methods (LP, MILP, MINLP, GDP and global optimization) can be coupled with single-objective multiobjective optimization approaches, such as aggregation methods, the epsilon constraint method, goal programming and goal attainment. Furthermore, parametric optimization techniques are especially attractive for efficiently obtaining Pareto-optimal solutions in linear bicriterion optimization problems (Pistikopoulos et al., 2007).

\section{Modeling Systems}

As described in the previous sections, the most common form of EWO models take the form of deterministic or stochastic MILP or MINLP models. Currently, it is possible to implement these models in commercial modeling systems since the models are expressed in equation form. This feature represents a significant advantage when compared for instance to process simulation, in which procedural approaches are common in large part due to the need of handling thermodynamic properties. Therefore, many planning, scheduling and supply chain models have been implemented in commercial modeling systems such as AIMMS, AMPL, GAMS and OPL. Furthermore, the effort involved is relatively modest.

All modeling systems allow the user to implement models in the form of algebraic models involving variables, discrete and continuous, and constraints and an objective function. Since it is possible to use indexed variables and constraints it is easy to implement models in compact form, similar to those expressed analytically, with which largescale problems are generated automatically by appropriate definition of sets for the indices (e.g. products, lines, time intervals) and corresponding data for the indexed parameters. Furthermore, a major advantage of these modeling systems is that they automatically interface with optimization solvers with which the user need not be concerned with low level programming details (e.g. MPS format, or supplying subroutines) to use optimization software. Moreover, for nonlinear models, these modeling systems perform automatic differentiation with which the user need not be concerned with supplying derivative information for the nonlinear solvers.

Finally, the modeling systems have capabilities of interfacing with spreadsheet, databases or graphics packages, thereby facilitating the deployment of the model as a tool with graphical user interface that can be used by nonspecialist users. Using these features, it is very easy to create new test instances and demonstrate the potential of optimization, as well as embed the solution into e.g. MS Office environment as the mathematical models are automatically propagated based on the given problem data. AIMMS has additionally graphical capabilities that allow easy implementation of prototype applications including Gantt chart displays, which in some cases may be sufficient 
for a final optimization solution. Other modeling environments can be connected to various visualization components, which often involve separate licensing costs.

Lastly, one important aspect is that models in these platforms have upward compatibility and are often platform independent (Windows, Linux, Unix, ...) which ensures their long term use, especially if they are properly documented. However, the compatibility across the modeling systems is restricted, as no detailed common modeling language standards exist.

\section{Major Issues}

As discussed in Grossmann (2005), and based on our experience with the EWO group at Carnegie Mellon, following are the major issues and challenges that arise in the application of the above described mathematical programming and modeling tools in EWO problems (we do not attempt here to provide a comprehensive review):

1. Linear versus Nonlinear Models. Many EWO problems are formulated as linear optimization models (mostly MILP), since the description of supply chains relies largely on simple inputoutput models, while scheduling models are usually expressed in terms of fixed production rates and processing times (Kondili et al., 2003; Pantelides, 2004; Sundaramoorthy and Maravelias, 2011). However, if for instance one wants to include stochastic inventory to determine safety stock (e.g. You and Grossmann, 2008), that gives rise to nonlinearities, and hence an MINLP problem. Furthermore, it is clear that applications that require a more detailed process description give rise to nonlinearities leading to MINLP models. A common case is for instance the handling of blending constraints that involve bilinearities, which are nonconvex (e.g. Mendez et al, 2006; Mouret et al., 2009). Another, common case arises in cyclic scheduling problems in which the objective must be divided by the cycle time, or when the inventory term must be integrated (e.g. Pinto and Grossmann, 1994). When dynamic process models are considered for optimizing non-regulated batch processes and their transitions (e.g. Bhatia and Biegler, 1996; Nystrom et al, 2005; Flores-Tlacuahuac and Grossmann, 2006), that gives rise to MIDO problems, which are often reformulated as complex MINLP models.

2. Multiscale Optimization. The spatial integration of geographically distributed manufacturing and inventory facilities in supply chains leads to large-scale problems that often require the application of specialized decomposition techniques (e.g. Graves, 1982; Gupta and Maranas, 1999; Jackson and Grossmann, 2003; Neiro and Pinto, 2006; Li and Ierapetritou, 2010). As discussed above, these usually involve Lagrangean decomposition, Benders decomposition, bi-level decomposition or rolling horizon algorithms. The temporal integration, however, requires effective representations and strategies in the first place so as to integrate long-term design decisions, with intermediate term production planning and shortterm scheduling decisions. These then require in turn decomposition schemes for the optimization across different time scales (e.g. Erdirik-Dogan and Grossmann, 2006; Liu et al., 2008; Vendarme and Floudas, 2008; Maravelias and Sung, 2009). A common example is rolling horizon strategies in which a detailed scheduling is recursively used in the first period, and an aggregate model in the subsequent time periods (e.g. Dimitriades et al., 1997; Lima et al., 2011). Finally, multisite planning and scheduling problems represent challenging problems as they require integration across both spatial and temporal scales (e.g. Terrazas-Moreno and Grossmann, 2011).

3. Optimization under Uncertainty. This issue is clearly pervasive in EWO problems. Uncertainties range from orders placed and equipment availability in scheduling problems, to uncertainties in prices and demands in large-scale supply chains. The former type of problems is generally best addressed through robust optimization techniques since the goal in short-term problems is largely the one of ensuring feasibility of the constraints over a given uncertainty range (e.g. Li and Ierapetritou, 2008). In contrast, for long-term strategic problems, stochastic programming is better suited because of its capability to account for recourse actions for the different scenarios, which is essentially equivalent to embedding a "quasi-simulation" in the optimization process. The most common formulation is the twostage programming model (e.g. Liu and Sahinidis, 1996; You et al., 2009). However, since most supply chain problem are multiperiod in nature, multistage stochastic programming is the natural formulation for these problems (e.g. Goel et al., 2006; Colvin and Marvelias, 2008; Tarhan et al, 2009). However, given the complexity of multistage problems, twostage programming models are usually used in order to make these problems manageable, and even then advanced computing architectures may be required (Linderoth and Wright, 2003).

4. Commercial vs. Off-the Shelf Software. In practice there is great temptation to use commercial software as opposed to developing tailor-made models and solution methods. Experience in our EWO group at Carnegie Mellon has shown that current off-the-shelf 
software is in fact rather limited. A major reason is that specific applications usually feature characteristics that make the problem inefficient for a more generic model (e.g. changeovers in discrete time STN or RTN models), or else require special treatment that is not contemplated in the general models (e.g. management of waste glass and its recycle). Perhaps the most useful software is for some generic planning problems (APO optimizer from SAP, PIMS from AspenTech) or for specialized scheduling applications (e.g. AspenOrion). Although the STN and RTN have given hope of developing generic scheduling software, the success of software such as VirtECS Scheduler and OSS has been rather limited. Therefore, a major trend has been to develop tailored models taking advantage of the flexibility and portability provided by modeling systems such as GAMS and AIMMS. Furthermore, the time for the development of these tailored models is quite reasonable and has the advantage of exploiting the particular features of the problem at hand. These modeling systems can also be readily interfaced with Excel spreadsheets and ERP software such as Oracle and SAP. It should also be mentioned that using as a basis generic models such as the RTN model has proved to be attractive to industry.

5. Solution of large-scale problems. While many EWO models, especially if they correspond to MILP problems, can in principle be solved with commercial optimization software (e.g. CPLEX, Gurobi and XPRESS), it is often the case that industrial applications lead to problems that are at least one order of magnitude larger than academic problems that are commonly used to test new models. This often poses the problem of either having to develop special purpose exact algorithms (Pekny et al., 1990; You et al, 2009), or else to develop solution strategies that are usually heuristic in nature, and are aimed at obtaining "good feasible solution" (e.g. Kelly, 2002; Castro et al, 2011). There is of course also the option of exploiting advanced computer architectures (Goux and Linderoth, 2003; Ferris and Munson, 2000).

\section{Illustrative examples}

In this section we present five examples that illustrate the major issues and challenges in the area of Enterprise-wide Optimization. Example 1 deals with a refinery planning model that involves nonlinear process model for the CDUs, illustrating the challenge of migrating from linear to nonlinear programming models. Example 2 deals with the scheduling and planning in production-distribution network of continuous multiproduct plants that involve different temporal and spatial scales. This example illustrates the challenge of multi-scale modeling given the dissimilar nature of the activities and the need of combining a detailed scheduling model with a high-level planning model. Example 3 deals with the design of tank sizes and distribution of industrial gases, which also illustrates another dimension of multiscale optimization problems. Example 4 deals with the design and planning of offshore oil field facilities with nonlinear reservoir models under uncertainty. This example illustrates the challenge of handling nonlinearities in multistage stochastic programming models. Finally, Example 5 deals with a supply chain design and planning for polymer manufacturing under the criteria of responsiveness and economics with the presence of demand uncertainty. This example illustrates the challenge of quantifying attributes like responsiveness and solving multiobjective optimization problems.

\section{Example 1}

Production planning modeling is an essential tool in the operation and management of modern refineries. It has traditionally relied on linear programming (LP) principles and methods for simple and robust planning models, such as the fixed-yield planning models and swing cuts planning models. However, these models fail to reflect the true nonlinear nature of the processing units. Alattas et al. (2011) have recently developed a fractionation index model (FI) to add nonlinearity to the linear refinery planning models. The FI model is developed as a more accurate nonlinear model for the complex crude distillation unit (CDU) than the fixed yield or the swing cuts models.

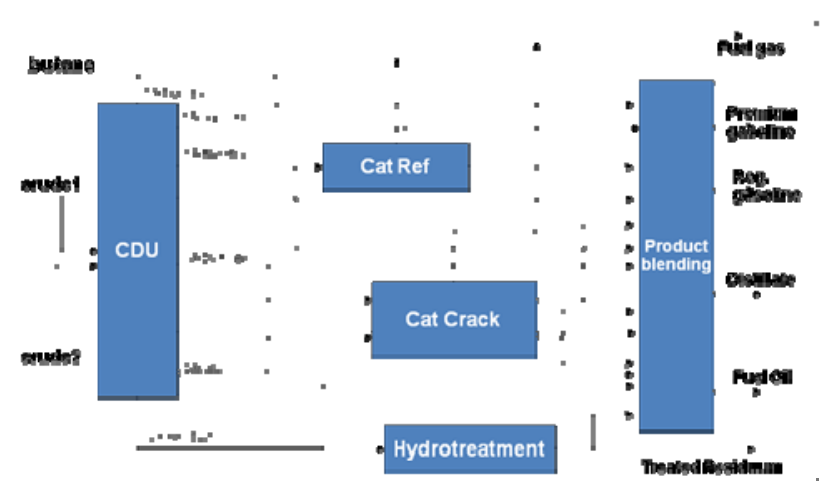

Figure 5. Complex refinery configuration

To illustrate the use of the FI-based planning model, the refinery configuration is shown in Figure 5. Three cases are analyzed where the refinery is processing 2 (Crudes 3, 4), 3 (Crudes 2, 3, 4) and 4 (Crudes 1, 2, 3, 4) types of crude oils, where Crude1 is the heaviest and Crude 4 is the lightest. The objective is to select the crude oils and quantities that will maximize refinery profit, while meeting the specified product demand and qualities. The available crude oil assays 
are used to generate the pseudo-components for use in the case studies. The detailed data are given in Alattas et al. (2011).

The FI model predicts the largest profit in all three cases, as seen in Table 1. The swing cut (SC) model also predicted profits that are much better than the basic FY model. In terms of crude oil feeds, the three models calculate different proportions of the available crude oils (Table 2). However, FY and FI preferred the middle crudes over the lightest or heaviest, using the middle Crude 3 in all cases while increasing the use of the similar but slightly lighter Crude 2 when introduced in Case 2 and Case 3 . However, the FI model consistently used less of Crude 3 than FY. SC, on the other hand, preferred the lightest crude, keeping the rest at the minimum.

Table 1 Refinery profits as calculated from the three planning models (\$1000/day)

\begin{tabular}{|c|c|c|c|}
\hline Iodal & Cond & $\cos 2$ & $\cos \theta$ \\
\hline F & 216 & 20 & 24 \\
\hline 80 & 195 & 195 & 191 \\
\hline$\Gamma \boldsymbol{\Gamma}$ & ot & 12 & $\omega$ \\
\hline
\end{tabular}

Table 2 Calculated crude oil feed rate (1000's bbl/day)

\begin{tabular}{|c|c|c|c|c|c|}
\hline$c=0$ & Whl & and & and & Cond- & Gond \\
\hline \multirow[t]{3}{*}{ low } & $\mathrm{Fr}$ & & & 4 & 6A \\
\hline & 6 & & & 10 & $\mathbf{m}$ \\
\hline & $\boldsymbol{n}$ & & & 20 & 72 \\
\hline \multirow[t]{3}{*}{ anon } & $\mathrm{Fr}$ & & 8 & 41 & 10 \\
\hline & $x$ & & 10 & 10 & E0 \\
\hline & $\boldsymbol{n}$ & & E0 & 30 & 10 \\
\hline \multirow[t]{3}{*}{ ond } & $\mathrm{Fr}$ & 10 & 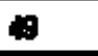 & 31 & 10 \\
\hline & $\infty$ & 10 & 10 & 10 & $\pi$ \\
\hline & $\boldsymbol{\pi}$ & 10 & B1 & 19 & 10 \\
\hline
\end{tabular}

In terms of the impact on the model size and solution time, the FI model is significantly larger, but does not require substantial increase in the solution time. The simplicity of the model is maintained by using the continuous Heaviside step function for the temperature cuts, which avoids the use of an MINLP formulation, although it has the potential drawback that it introduces nonconvexities. The models statistics are shown in Table 3. All three models were implemented in GAMS and used the CONOPT 3.14 solver for the FI model and CPLEX 11.1.1 solver for the linear FY and SC models.

\section{Example 2}

Terrazas-Moreno and Grossmann (2011) recently addressed the solution of simultaneous scheduling and planning problems in production-distribution networks (Fig. 6) of continuous multiproduct plants that involve different temporal and spatial scales. Production planning results in medium- and long-term decisions, whereas production scheduling determines the timing and sequence of operations in the short-term. The production-distribution network is made up of several production sites distributing to different markets. The planning and scheduling model has to include spatial scales that go from a single production unit within a site, to a geographically distributed network. TerrazasMoreno and Grossmann (2011) proposed two decomposition methods to solve this type of problems. One method corresponds to the extension of the bi-level decomposition of Erdirik-Dogan and Grossmann (2008) to multi-site, multimarket networks. A second method is a hybrid decomposition method that combines bi-level and spatial Lagrangean decomposition methods.

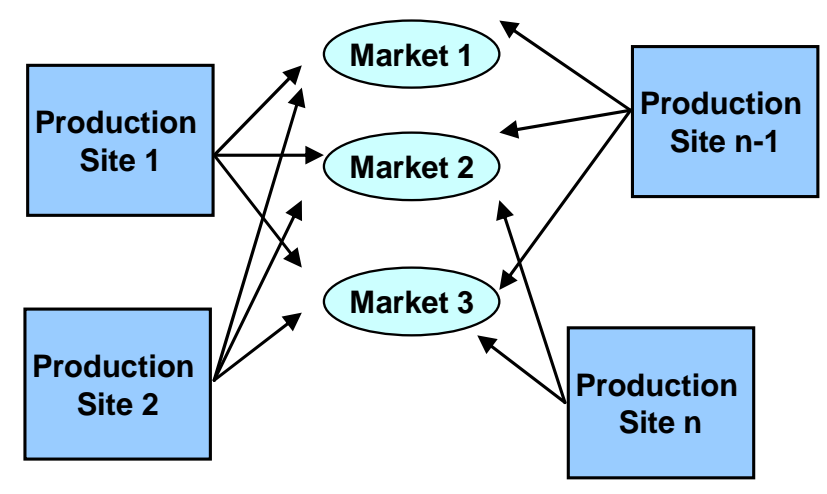

Figure 6. Production and distribution network

As an illustration consider a small manufacturing and distribution network that consists of 3 production sites with single production lines serving 2 markets that demand products $\mathrm{A}, \mathrm{B}$, and $\mathrm{C}$. The objective is to obtain the optimal planning and detailed production schedule for 4 weeks. Data on the maximum production rates in each site, values of market demand for all products, while transition times and costs can be found in Terrazas-Moreno and Grossmann (2011). 
Table 3 Models statistics

\begin{tabular}{|c|c|c|c|c|c|}
\hline Case & Model & Yartabs: & Equations & 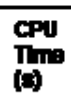 & Monlear \\
\hline \multirow[t]{3}{*}{ Cased 1} & $F^{\prime \prime}$ & 140 & t50 & 6.7 & \\
\hline & $s c$ & 103 & 10 & 7.3 & \\
\hline & $\boldsymbol{F}$ & 1225 & 2004 & 7.3 & 3*0 \\
\hline \multirow[t]{2}{*}{$C=002$} & $\begin{array}{l}F^{\prime} \\
S c\end{array}$ & $\begin{array}{l}105 \\
215\end{array}$ & $\begin{array}{l}61 \\
176\end{array}$ & $\begin{array}{l}0.4 \\
9.0\end{array}$ & \\
\hline & F & 1008 & $17 / 2$ & 808 & 522 \\
\hline \multirow[t]{3}{*}{$C=03$} & $F^{\prime \prime}$ & 201 & tal & 98 & \\
\hline & $\mathrm{sc}$ & 71 & 214 & 10.3 & \\
\hline & F & 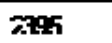 & 200 & Iกค & F96 \\
\hline
\end{tabular}

This example is a small case study so it is possible to obtain an optimal solution without decomposition techniques. The full space model consists of 144 discrete variables, 1117 continuous variables, and 1303 constraints. Using CPLEX 12.2.0 in GAMS version 23.6 for Windows, using an Intel Core i7 CPU at $2.93 \mathrm{GHz}$, and $4.00 \mathrm{~GB}$ of RAM requires less than $1 \mathrm{CPU}$ sec. Figure 7 shows the Gantt chart that corresponds to the optimal production schedule for every site, which leads to a profit of $\$ 69,711$. Product $\mathrm{A}$ is assigned more production time since it is the most profitable. Note that after week 2 each site is dedicated to a single product.

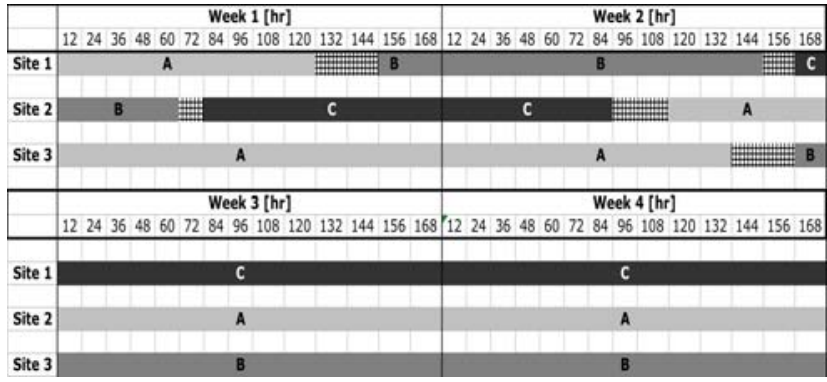

Figure 7. Optimal production schedule

In examples, with up to 6 sites, 25 products and 24 weeks, the computational performance of the hybrid algorithm was similar to bi-level decomposition in medium-sized problems and faster in large-scale problems. In all case studies (see Fig. 8) the hybrid algorithm converged within a $2 \%$ of the optimality tolerance. This method is therefore efficient for problems with tens of thousands of discrete variables, and hundreds of thousands of continuous variables and constraints.

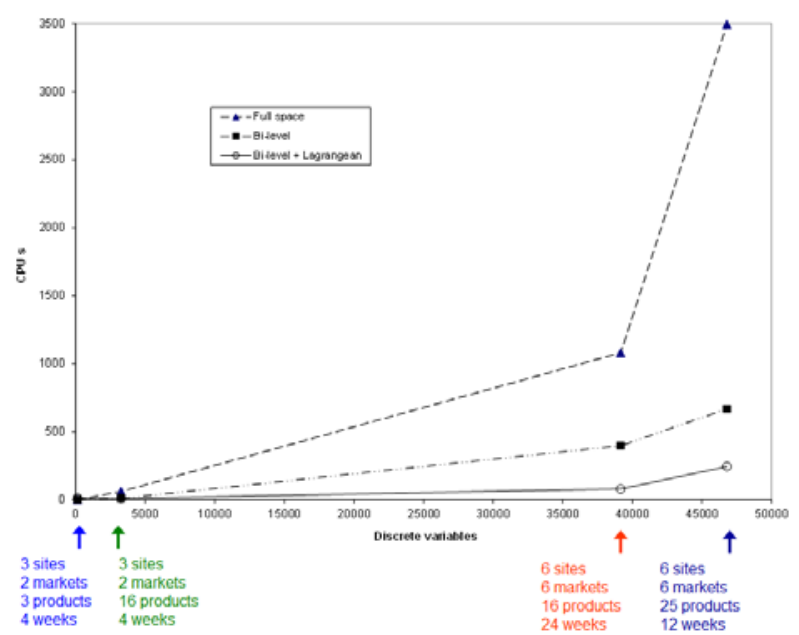

Figure 8. Solution time for case studies using full space, bi-level, and hybrid algorithms

\section{Example 3}

You et al. (2011) addressed the optimization of industrial gas distribution systems, which consist of plants and customers, as well as storage tanks, trucks and trailers. The specific problem assumes an industrial gas distribution network consisting of a production plant and a set of customers as shown in Figure 9. The locations of the plant and customers, as well as the distances between them are given. Each customer has a deterministic and constant demand rate and safety stock level in each year within the planning horizon. Given is also a set of possible tanks with different discrete sizes. For customers with existing tanks, their specific initial inventory levels are given. New customers need to determine the size of tank to be installed in their location. Existing customers can upgrade or downgrade the existing tanks, or add a second tank if extra space is available. There are a numbers of trucks of size with discrete capacities. The delivery cost per distance traveled for every truck is also given. All the trucks are assumed to have the same average traveling speed and a maximum number of working hours per day.

You et al. (2011) presented a simultaneous MILP model to minimize the total capital and operating cost, and to integrate short-term distribution planning decisions for the vehicle routing with long-term inventory decisions for sizing storage tanks at customer locations. To circumvent the largescale MILP model, these authors proposed a strategy that employs a continuous approximation (Langevin et al., 1996) to estimate the annual delivery cost without considering the detailed schedules of the routing problem. By accounting for the capacitated vehicle routing cost at the strategic level, the trade-off between the capital cost and operational cost is established. After the strategic tank sizing decisions are determined, detailed vehicle routing is considered for operational decisions. The major advantage is that both the upper level continuous approximation model and the lower 
level detailed routing problem can be solved effectively without sacrificing too much solution quality.

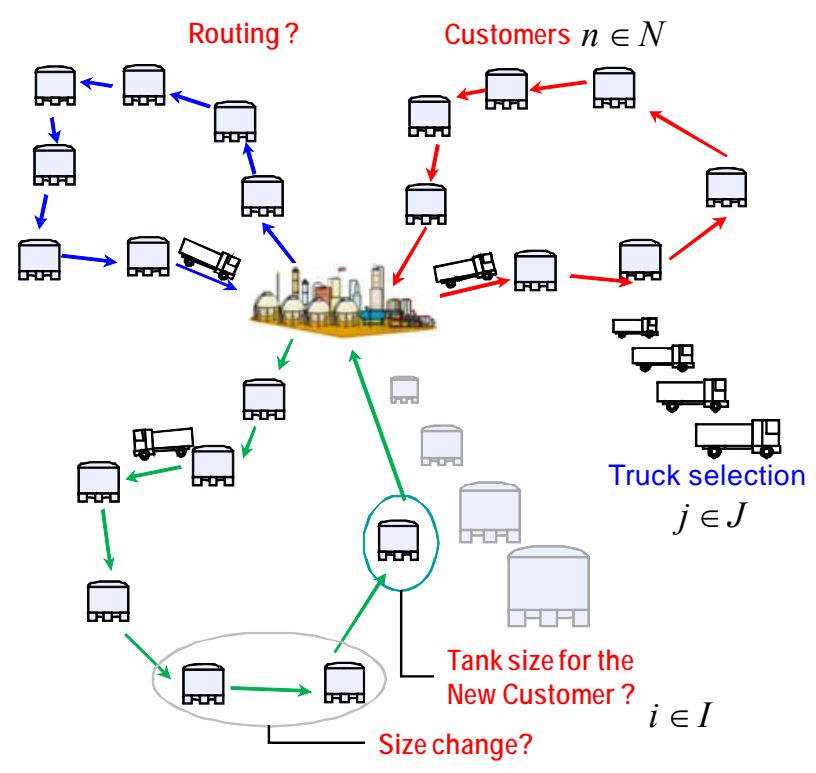

Figure 9. Tank sizing and vehicle routing of industrial gas supply chains

As a specific example, consider a four-customer industrial gas cluster, of which the network structure and the demand rates of the first year are given in Figure 10. Based on this network, there are 15 possible routes for this case study. Also, from the network structure, it is easy to see that the TSP distance to visit all the customers once is $50877 \mathrm{~km}$ for this case study.

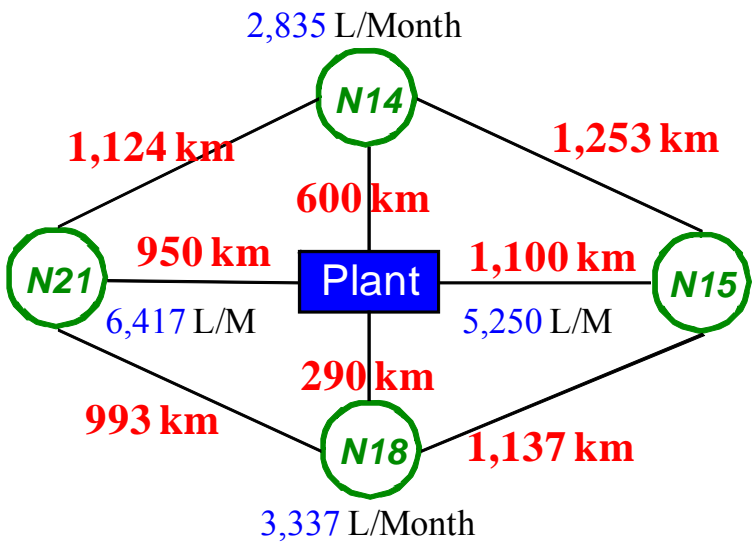

Figure 10. Four-customer industrial gas supply chain

Table 4 Optimal solution of the second instance of case study 2 (three year planning horizon, and N14,

\begin{tabular}{|c|c|c|c|}
\hline & \multirow{2}{*}{ 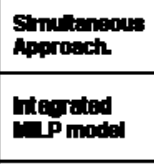 } & \multicolumn{2}{|c|}{$\begin{array}{l}\text { Conthuous Approstmetion } \\
\text { Approesch }\end{array}$} \\
\hline & & $\begin{array}{l}\text { Conthuous: } \\
\text { approutunatlo } \\
\text { n model }\end{array}$ & $\begin{array}{l}\text { Dotedled } \\
\text { rocthe model }\end{array}$ \\
\hline Dis. Var. & $10,8+\theta$ & 79 & 10,800 \\
\hline Cont Var. & 18,440 & 578 & 18,08 \\
\hline Constrahts & 23,834 & 723 & 23814 \\
\hline CPU $(x)$ & $\begin{array}{l}51,406 \\
\text { Prmamasos }\end{array}$ & 5.3 & 31,420 \\
\hline Tolvel and & 19574: & \multicolumn{2}{|l|}{$\$ 101,00$} \\
\hline Propoped lankd & 19,000: & \multicolumn{2}{|l|}{$10,000 \mathrm{~L}$} \\
\hline 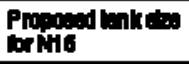 & 20,000/ & \multicolumn{2}{|l|}{ 10,000 L } \\
\hline 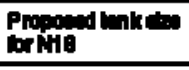 & 10,000: & \multicolumn{2}{|l|}{$100000 \mathrm{~L}$} \\
\hline 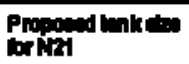 & 20000 & \multicolumn{2}{|l|}{$200000 \mathrm{~L}$} \\
\hline
\end{tabular}

The planning horizon is 3 years and all customers are treated as new without any existing tanks. In the three-year horizon, a $15 \%$ demand growth rate is considered for all customers. The computational results are given in Table 4 . We can clearly see that the problem sizes increase significantly and some problems include more than 10,000 binary variables. The simultaneous approach ran out of memory after around 4 hours, and the best solution found $(\$ 156,774)$ has a gap as large as $74.54 \%$. Because there are 4 customers and 15 possible routes, the problem size of the route selection - tank-sizing model also becomes computationally intractable.

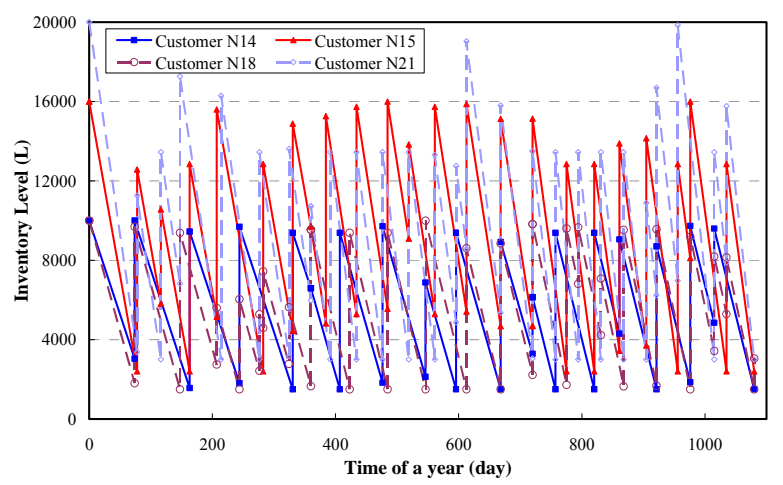

Figure 11. Optimal inventory profiles of the four customers in the second instance of case study 2 (three year planning horizon, and N14, N15, N18 and $N 21$ are all new customers)

With the continuous approximation approach, the problem size of the upper level approximation model is rather small and can be solved very efficiently (only $5.3 \mathrm{~s}$ for the global optimum). The detailed routing model with fixed tank sizes, despite its large size, was solved to global optimality in about 9 hours. The solution predicted by this 
approach has a lower total cost of $\$ 101,402$, and the optimal tank sizes for customers N14, N15, N18 and $\mathrm{N} 21$ are 10,000L, 16,000L, 10,000L and 20,000L, respectively. The detailed inventory profiles are given in Figure 11, that account for the trade-offs between tank sizes, demand rates and deliveries.

\section{Example 4.}

Tarhan et al. (2009) considered the design and planning of an offshore oil field infrastructure under uncertainty over a specified planning horizon (Fig. 11). The main uncertainties considered were in the initial maximum oil or gas flowrate, recoverable oil or gas volume, and water breakthrough time of the reservoir, which are represented by discrete distributions. Furthermore, it was assumed that these uncertainties are not immediately realized, but are gradually revealed as a function of design and operation decisions. Specifically, the authors considered a field consisting of several reservoirs where a number of wells can be drilled and exploited for oil in every reservoir during the planning horizon. The field infrastructure can be composed of Floating Production Storage and Offloading (FPSO) (Fig. 12) and/or Tension Leg Platform (TLP) (Fig. 13) facilities. The FPSO facility can be either a small FPSO, converted from a retiring oil tanker, or a large FPSO, a newly constructed grassroots facility. An FPSO facility can process, store and offload the processed oil to other tankers. Unlike FPSO, a TLP facility cannot process oil; it possesses only drilling and oil recovering capability. TLP and FPSO facilities can be connected to each other through risers and sub-sea pipelines. There are two options for drilling wells. Each well can be drilled either as a sub-sea or a TLP well. Drilling ships are used to drill sub-sea wells, so there is no need to have a facility present to drill a sub-sea well. Unlike sub-sea wells, a TLP well has to be drilled by a TLP facility.

The problem involves making investment and operation decisions over the planning horizon. Investment decisions are selection of the number, type and capacity of facilities and installation schedule of these facilities, as well as selection of types of wells and drilling schedule of wells. Operation decisions are amount of oil production for each time period given the limitations of the reservoirs. The goal was to maximize the expected net present value of the project

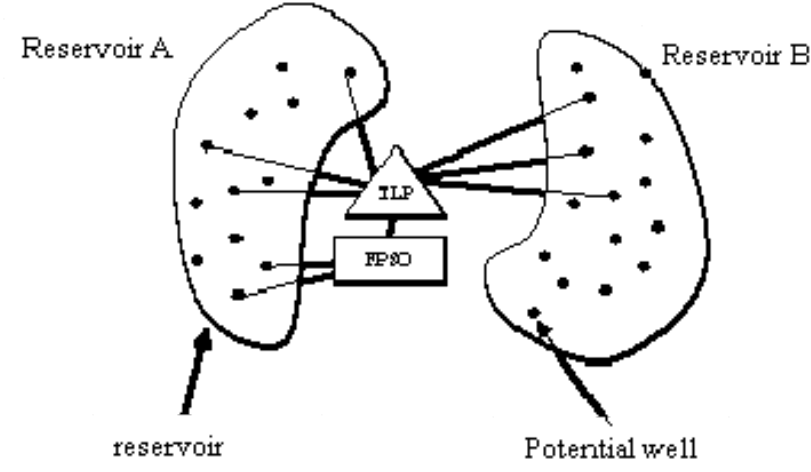

Fig. 11: A Typical oil field infrastructure.

In order to account for these decision-dependent uncertainties, Tarhan et al. (2009) proposed a multistage stochastic programming model that incorporates nonlinear reservoir behavior. The proposed solution algorithm relies on a duality-based branch-and-bound method involving subproblems as nonconvex mixed-integer nonlinear programs that are solved to global optimality with BARON (Tarhan et al., 2009).

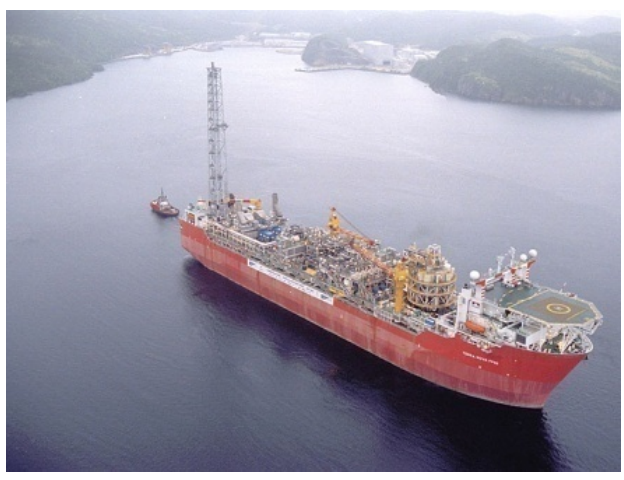

Figure12. A typical FPSO facility 


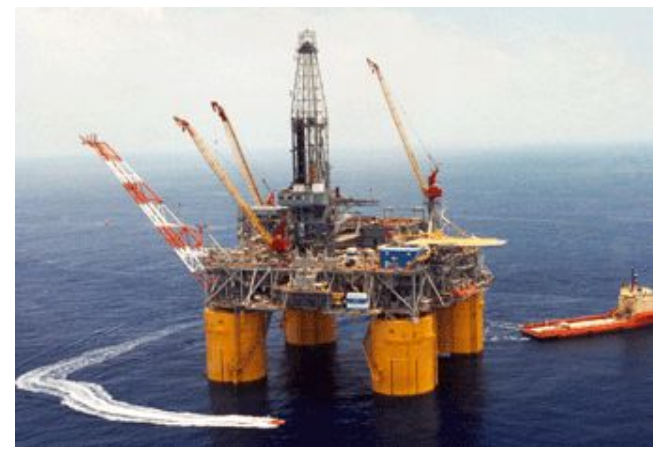

Figure 13. A typical TLP facility

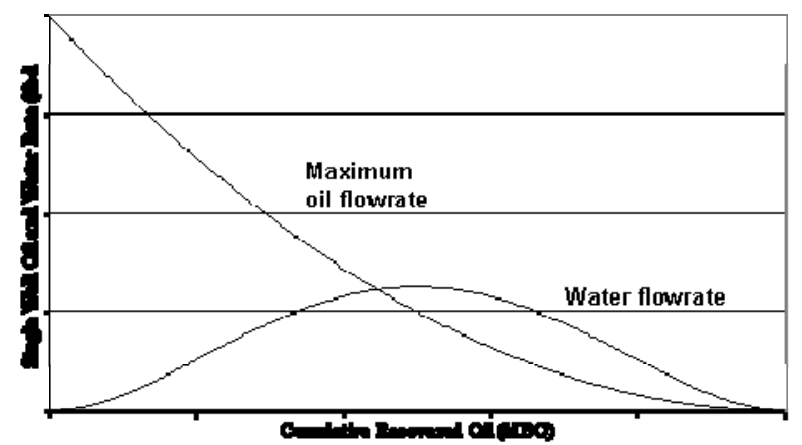

Figure 14. Nonlinear reservoir model

In one of their examples, Tarhan et al (2009) considered an offshore oil field having a single reservoir over a 10-year horizon. The uncertainties in initial maximum oil flowrate, the size of the reservoirs and water breakthrough time were represented by discrete distributions consisting of two values (high and low). These uncertainties were incorporated into the model using eight different scenarios in Table 5.

\section{Table 5: Representation of scenarios using uncertain parameter}

\begin{tabular}{|c|c|c|c|c|c|c|c|c|}
\hline & \multicolumn{8}{|c|}{ 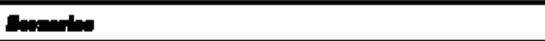 } \\
\hline & 1 & 2 & $a$ & 4 & 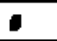 & 0 & 1 & 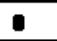 \\
\hline 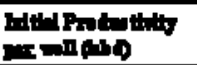 & ט0 & u & $\mathbf{n}$ & $\mathbf{n}$ & 10 & ic & $\boldsymbol{\eta}$ & $\boldsymbol{n}$ \\
\hline 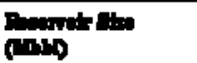 & 300 & 30 & 300 & 303 & 0.5 & 120 & 1000 & 190 \\
\hline 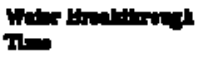 & 5 & 2 & s & 2 & 3 & 2 & 3 & 2 \\
\hline
\end{tabular}

Given the eight scenarios over the 10-year horizon, the expected value solution using mean values proposes building 4 small FPSOs, 3 TLP in the first year and start drilling 12 TLP wells in year 2. These decisions resolve the uncertainty in initial productivity and size in year 3 . The production starts at year 3 and after all the scenarios become distinguishable at year 4 , different decisions are implemented for the rest of the time horizon to maximize the net present value. This expected value approach gives an objective function value of $\$ 3.76 \times 10^{9}$. The optimal multistage stochastic programming solution yields an expected net present value of $\$ 4.59 \times 10^{9}$ which is $22 \%$ higher profit than the expected value solution. The solution proposes building 2 small FPSO, 1 TLP facility and drilling 9 sub-sea wells initially. Drilling these 9 wells will resolve the uncertainty in initial oil flowrate and reservoir size. In year 2, for scenarios 1 and 2 there is no further investment, while in scenarios 3 and 43 TLP wells are drilled. For scenarios (5-6) the solution proposes building 4 small FPSO facilities, 5 TLP facilities and drilling 12 sub-sea wells. For scenarios (7-8), the solution proposes building 4 small FPSO facilities, 3 TLP facilities and drilling 6 TLP wells and 6 sub-sea wells.

Fig.15 compares the net present values of the expected value solution (columns in pattern) and the stochastic programming solution (columns in black) over the 8 scenarios in the 10 year horizon. As it can be seen the expected value approach can lead to losses in two of the scenarios. Thus, stochastic programming not only generates solutions with higher expected net present values, but also more robust solutions since in all cases the NPV is positive.

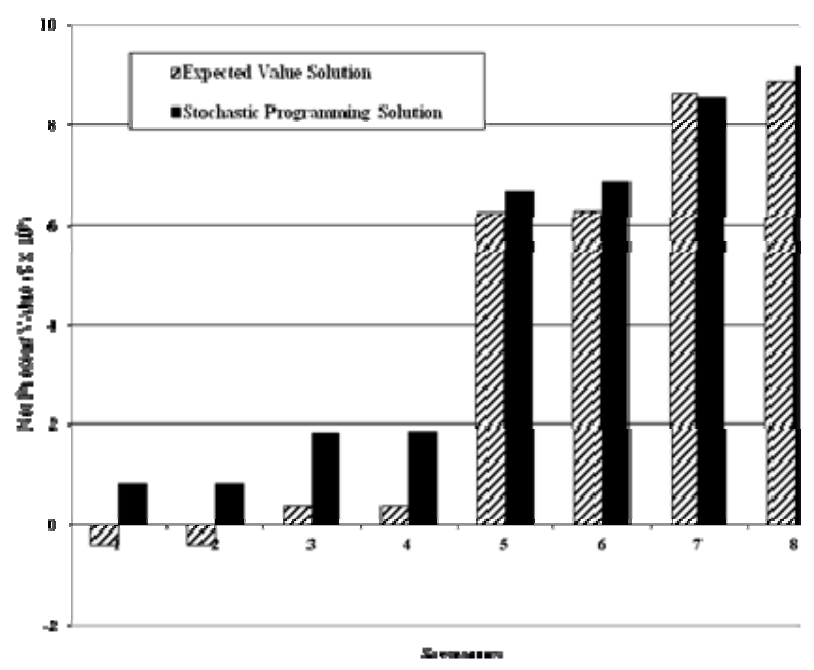

Figure 15. Comparison of net present values

The corresponding MINLP model involved 800 0-1, 800 integer variables, 869 continuous variables and 8088 constraints. The best feasible solution proposed by the algorithm after 120 hours is guaranteed to be within $12 \%$ optimality gap. A total of 14 nodes were traversed and the best feasible solution was found after 90 hours at Node 6 of the duality-based branch-and-bound method. The CPU time was subsequently reduced to 5 hours with a strategy that reduced the number of times a global optimization problem had to be solved (Tarhan et al, 2010).

\section{Example 5}

You and Grossmann (2008) addressed the optimization of supply chain design and planning under the criteria of responsiveness and economics with the presence of demand 
uncertainty. The supply chain consists of multi-site processing facilities and corresponds to a multi-echelon production network with both dedicated and multiproduct plants. The economic criterion was measured in terms of net present value, while the criterion for responsiveness accounts for transportation times, residence times, cyclic schedules in multiproduct plants, and inventory management. By using a probabilistic model for stockout, the expected lead time was proposed as the quantitative measure of supply chain responsiveness. The probabilistic model can also predict the safety stock levels by integrating stockout probability with demand uncertainty. These were all incorporated into a multi-period mixed-integer nonlinear programming (MINLP) model, which takes into account the selection of manufacturing sites and distribution centers, process technology, production levels, scheduling and inventory levels. The problem was formulated as a bi-criterion optimization model that maximizes the net present value and minimizes the expected lead time. The model was solved with the $\varepsilon$ constraint method and produces a Pareto-optimal curve that reveals how the optimal net present value, supply chain network structure and safety stock levels, change with different values of the expected lead time.

You and Grossmann (2008) considered a large scale problem that was motivated by a real world application in which various locations for suppliers, plants, distribution centers and customers were considered as seen in Figure 16. The plants involve a dedicated plant for producing styrene (Plant I), and two plants for producing two grades of solid polystyrene (Plant II) and three grades of expandable polystyrene (Plant III). The potential supply chain network (Figure 17) includes three possible ethylene suppliers located in Illinois, Texas and Mississippi, and three potential benzene suppliers located in Texas, Louisiana, Alabama. Four potential manufacturing sites can be located in Michigan, Texas, California and Louisiana. The Michigan manufacturing site can set up all the three types of plants, the Texas manufacturing site can only install Plant I, the California manufacturing site can only set up Plants II and III, and the Louisiana manufacturing site can only set up Plants I and II. The supply chain can have five distribution centers, located in Nevada, Texas, Georgia, Pennsylvania and Iowa. Customers are pooled into nine sale regions across the country based on their geographical proximity. The corresponding superstructure of the supply chain network is given in Figure 17.

The problem was solved assuming that the demands follow triangular distributions, and with consideration of safety stock and without it. Both instances consist of 215 binary variables, 8,216 continuous variables and 14,617 constraints and they were solved with a bi-level decomposition algorithm by using GAMS/BARON. Six points in the Pareto optimal curve require 15,396 CPU seconds for Instance 1 (with safety stock) and 16,927 seconds for Instance 2 (without safety stock). The Pareto optimal curves are given in Figure 18. The Pareto curve ranges from 1.6 to 5 days in the expected lead time and from $\$ 409$ million to $\$ 683$ million for NPV. The Pareto curve without safety stock) ranges from 4.3 to 5 days. This example shows the importance of establishing trade-offs between responsiveness and economics in the design and planning of a process supply chain network.

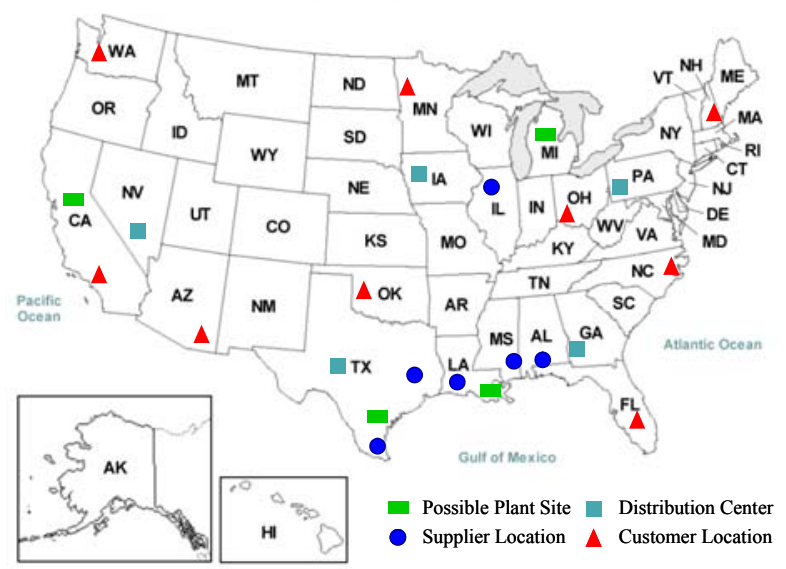

Figure 16. Location map for Example 5

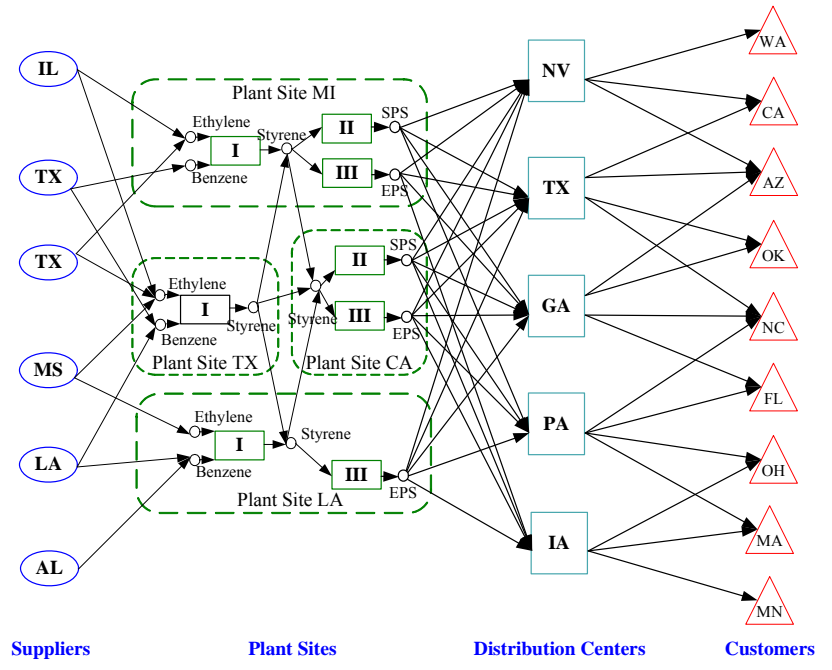

Figure 17. Potential process supply chain network superstructure for Example 2

\section{Concluding Remarks}

This paper has provided an overview of the area of Enterprise-wide Optimization that is driven by needs of the process industries for reducing costs and remaining competitive in the global marketplace. A brief review was presented of the relevant mathematical programming techniques. Some of the major issues and challenges in their application to EWO problems have been highlighted, and 
several examples presented to illustrate the nature of the applications and the problems that are faced.

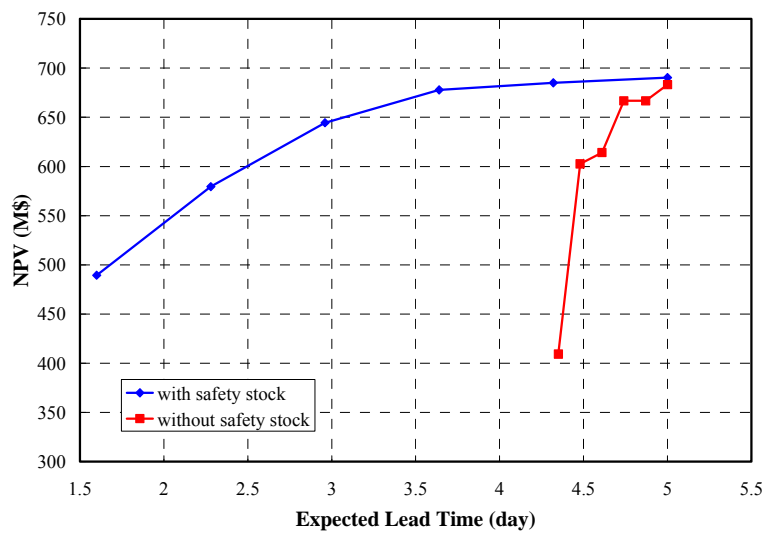

Figure 18. Pareto curve for Example 2 with safety stock and without safety stock (Instance 1 and 2 of Example 2)

It is hoped that this paper has shown that EWO has become a reality and that has industrial relevance. Nevertheless, it is clear as was discussed, that some of the major issues and challenges involve handling of nonlinearities, multiscale temporal and spatial integration, anticipating uncertainties, selecting between commercial vs. tailored solutions, and effective solution of industrial-sized problems. In addition to these issues, there are three major outstanding challenges that remain to be addressed and are worth of future research efforts. In particular:

Optimizing entire supply chains. It is clear that while important progress has been made in modeling and optimizing major components of supply chains, the optimization of entire supply chains still remains an elusive problem, especially if the objective is to integrate planning with scheduling in these supply chains. The difficulty is in part due to the very large size of the resulting models. But it is in fact also due to the somewhat distinct nature of the major components in a supply chain. For instance, in the petroleum supply chain it is not be very obvious how to integrate the models for upstream exploration, marine transportation, crude oil delivery unloading and refinery optimization since they often rely on different representations (Kelly, 2006; Shah et al., 2011).

Integrating control with planning and scheduling. While the pyramid of decision-making in process operations contains the three elements, it is clear that most of the attention has been devoted to the integration of planning and scheduling, there is still relatively little work that has been reported on the integration of these activities with control (e.g. Bhatia and Biegler, 1996; Perea et al., 2003). While conceptually this should be a logical extension (Harjunkoski et al., 2009), major barriers are the complexity and large size of the resulting MIDO optimization problem, which will require significant progress for effective solution methods.

Design and operation of sustainable supply chains. Sustainability is clearly becoming a major concern in the process industries, and therefore it would be natural to consider it as part of EWO. As pointed by Hugo and Pistikopoulos (2005) mathematical programming methods have been employed to account for environmental concerns and ecological footprint in process design problems that focus on single plants. However, their application to the entire supply chain networks under a multi-objective optimization framework has been rather limited. A recent review on this topic can be found in Grossmann and GuillenGozalbez (2010), which clearly indicates scope for further work in this area.

Finally, it is hoped that this article will promote interest in addressing the outstanding challenges in EWO. It is clear that the challenges are non-trivial, but the payoff is that it is highly relevant as it can greatly help to strengthen the process industry.

\section{Acknowledgment}

The author would like to acknowledge financial support from the member companies of the Center of Advanced Process Decision-making at Carnegie Mellon.

\section{References}

Abhishek K., Leyffer S. and Linderoth J.T., FilMINT: An OuterApproximation-Based Solver for Nonlinear Mixed Integer Programs, ANL/MCS-P1374-0906, Argonne National Laboratory, 2006.

Achterberg T., Koch T., Martin A., 2006, MIPLIB 2003. Operations Research Letters. 34(4), 361-372.

Adams, J., E. Balas and D. Zawack, "The Shifting Bottleneck Procedure for Job Shop Scheduling," Management Science, 34:391-401 (1988).

Alattas, A., I.E. Grossmann and I. Palou-Rivera, "Integration of Nonlinear CDU Models in Refinery Planning Optimization," Ind. Eng Chem. Res. 50, 6860-6870 (2011).

Balas, E. (1979). Disjunctive Programming. Annals of Discrete Mathematics, 5:3-51.

Bassett M. H., Pekny, J.F., Reklaitis, G.V., "Decompositon Techniques for the Solution of Large-scale Scheduling Problems," AIChE J., 42, 3373 (1996).

Beaumont N., An Algorithm for Disjunctive Programs, European Journal of Operational Research, 48, 362-371, (1991).

Belotti P., Lee J., Liberti L., Margot F. and Wachter A., Branching and Bounds Tightening Techniques for Non-convex 
MINLP, Optimization Methods and Software, 24:4, 597-634, 2009

Benders J. F. (1962). Partitioning Procedures for solving mixed variables programming problems. Numersiche Mathematik, 4, 238 - 252

Ben-Tal, A.; El Ghaoui, L.; Nemirovski, A. (2009) Robust Optimization, Princeton University Press.

Bertsimas, D.; Sim, M. Robust Discrete Optimization and Network Flows, Mathematical Programming, 98, 49, (2003).

Bhatia, T., and Biegler, L.T., "Dynamic Optimization in the Design and Scheduling of Multiproduct Batch Plants," I \& EC Research, 35, 7, p. 2234 (1996).

Biegler, L. T. Nonlinear Programming: Concepts, Algorithms and Applications to Chemical Processes, SIAM, Philadelphia (2010)

Birge, J. R., Louveaux, F. (1997). Introduction to Stochastic Programming. New York, NY: Springer.

Bixby R., Rothberg E, 2007, Progress in Computational Mixed Integer Programming- a Look Back from the Other Side of the Tipping Point, Annals of Operation Research, 49(1), 37-41.

Bixby R.E., 2002, Solving Real-world Linear Programs: a Decade and More of Progress. Operations Research, 50(1), 3-15.

Bok, J.-K.; Grossmann, I. E.; Park, S., Supply Chain Optimization in Continuous Flexible Process Networks. Industrial \& Engineering Chemistry Research, 39, (5), 1279-1290, (2000).

Bonami, P., L.T. Biegler, A.R. Conn, G. Cornuejols, I.E. Grossmann, C.D. Laird, J. Lee, A. Lodi, F. Margot, N. Sawaya, A. Wächter (2008) An Algorithmic Framework for Convex Mixed Integer Nonlinear Programs, Discrete Optimization 5, 186-204.

Brooke, A., D. Kendrick, A. Meeraus and R. Raman, "GAMS: A User's Guide”, GAMS Development Corporation, Washungton, DC (1998).

Castro, P.M., I. Harjunkoski and I.E. Grossmann, "Greedy Algorithm for Scheduling Batch Plants with Sequence-Dependent Changeovers," publication AIChE J. 57, 373-387 (2011).

Colvin, M., and C.T. Maravelias, "A stochastic programming approach for clinical trial planning in new drug development. Computers and Chemical Engineering 32, 2626-2642 (2008).

Deb, K. (2008). Multi-objective Optimization Using Evolutionary Algorithms. John Wiley \& Sons Inc.

Dimitriadis, A.D., Shah, N. and Pantelides, C.C., "RTN-Based Rolling Horizon Algorithms for Medium Term Scheduling of Multipurpose Plants," Computers \& Chemical Engineering, 21, S1061, (1997).
Duran, M. A.; Grossmann, I. E., “An Outer-approximation Algorithm for a Class of Mixed-integer Nonlinear Programs," Mathematical Programming, 36, 307 (1986).

Ehrgott, M. Multicriteria optimization. Springer (2000)..

Erdirik-Dogan, M. E.; Grossmann, I. E., “A Decomposition Method for the Simultaneous Planning and Scheduling of Singlestage Continuous Multiproduct Plants." Industrial \& Engineering Chemistry Research, 45, (1), 299 (2006).

Erdirik-Dogan M. and I. E. Grossmann "Simultaneous Planning and Scheduling of Single-stage Multiproduct Continuous Plants with Parallel Lines," Computers and Chemical Engineering, 32, 2664 - 2683 (2008).

Ferris, M. C., T. S. Munson. «Modeling languages and Condor: Metacomputing for optimization." Mathematical Programming 88 487-506 (2000).

Ferris, M.C., S. P. Dirkse, J.-H. Jagla, and A. Meeraus. "An extended mathematical programming framework," Computers and Chemical Engineering, 33:1973-1982, (2009).

Fletcher R. and Leyffer S., "Solving Mixed Integer Nonlinear Programs by Outer Approximation," Math Programming, 66, 327 (1994).

Flores-Tlacuahuac, A. and I.E. Grossmann, "Simultaneous Cyclic Scheduling and Control of a Multiproduct CSTR," Ind. Eng. Chem. Res., 45, 6698-6712 (2006).

Floudas, C.A.; Lin, X., "Continuous-time Versus Discrete-time Approaches for Scheduling of Chemical Processes: a Review," Computers and Chemical Engineering, 28, $2109-2129$ (2004).

Geoffrion A. M., "Generalized Benders Decomposition,” JOTA 10, 237-260 (1972).

Geoffrion A. M., Lagrangean Relaxation for Integer Programming," Mathematical Programming Study, 2, 82 - 114 (1974).

Goel, V., Grossmann, I. E., El-Bakry, A. S., Mulkay, E. L., “A Novel Branch and Bound Algorithm for Optimal Development of Gas Fields under Uncertainty in Reserves," Computers and Chemical Engineering, 30, 1076-1092 (2006).

Goux, J.-P. and Leyffer, S., "Solving Large MINLPs on Computational Grids," Optimization and Engineering, 3, 327-354 (2003).

Graves, S, "Using Lagrangean Techniques to Solve Hierarchical Production Planning Problems," Management Science, 28, 260-275 (1982).

Grossmann, I.E., "Review of Non-linear Mixed Integer and Disjunctive Programming Techniques," Optimization and Engineering 3, 227-252 (2002).

Grossmann, I.E. "Enterprise-wide Optimization: A New Frontier in Process Systems Engineering," AIChE Journal, 51, 18461857 (2005). 
Grossmann, I.E. and G. Guillen-Gosalbez, "Scope for the Application of Mathematical Programming Techniques in the Synthesis and Planning of Sustainable Processes," Computers and Chemical Engineering 34, 1365-1376 (2010).

Guignard M., Lagrangean Relaxation. “Top, 11(2), 151 - 228 (2003).

Gupta, A. and Maranas, C.D., "A Hierarchical Lagrangean Relaxation Procedure for Solving Midterm Planning Problems," Ind. Eng. Chem. Res, 38, 1937-1947 (1999).

Harjunkoski, I., R. Nystrom and A. Horch, "Integaration of Scheduling and Control: Theory or Practice," Computers \& Chemical Engineering, 33, 1909-1918, (2007).

Hooker, J.N., Logic-Based Methods for Optimization: Combining Optimization and Constraint Satisfaction, Wiley (2000).

Hooker, J.N., "Logic, optimization and constraint programming," INFORMS Journal on Computing 14 295-321 (2002)

Hooker, J.N. "Planning and scheduling by logic-based Benders decomposition, Operations Research 55, pp. 588602 (2007)

Ierapetritou, M.G. \& Floudas, C.A., "Effective Continuoustime Formulation for Short-term Scheduling. 1. Multipurpose Batch Processes," Industrial and Engineering Chemistry Research, 37, 4341 - 4359 (1998).

Ierapetritou M.G., J. Acevedo, and E.N. Pistikopoulos "An Optimization Approach for Process Engineering Problems under Uncertainty," Computers and Chemical Engineering, 20, 703 - 709 (1996).

Ierapetritou, M. G., Pistikopoulos, E. N., "A Novel Optimization Approach of Stochastic Planning Models," Industrial and Engineering Chemistry Research, 33, 1930-1942 (1994).

Iyer, R. R., Grossmann, I. E., "A Bilevel Decomposition Algorithm for Long-Range Planning of Process Networks," Industrial \& Engineering Chemistry Research, 37, 474-481 (1998).

Jackson J. R. and I. E. Grossmann, "Temporal Decomposition Scheme for Nonlinear-Multisite Production Planning and Distribution Models," Industrial and Engineering Chemistry Research, 42(13), 3045 3055 (2003).

Jain, V. and I.E. Grossmann, "Algorithms for Hybrid MILP/CP Models for a Class of Optimization Problems, ", INFORMS Journal of Computing, 13, 258-276 (2001).

Johnson, E.L., G.L. Nemhauser and M.W.P. Savelsbergh, "Progress in Linear Programming Based Branch-
and-Bound Algorithms: Exposition," INFORMS Journal of Computing, 12 (2000).

Jonsbraten, T.W., 1998. Optimization Models for Petroleum Field Exploitation. Ph.D. thesis, Norwegian School of Economics and Business Administration.

Jonsbraten, T.W., Wets, R.J. B., Woodruff, D.L., 1998. A Class of Stochastic Programs with Decision Dependent Random Elements. Annals of Operations Research 82, 83-106.

Kelly, J.D.., "Chronological decomposition heuristic for scheduling: divide and conquer," AIChE J., 48, 29952999 (2002).

Kelly, J.D., "Logistics The missing link in blend scheduling optimization," Hydrocarbon Processing, 85, p.45 (2006).

Kondili, E., Pantelides, C. C.; Sargent, R., "A General Algorithm for Short-Term Scheduling of Batch Operations - I. MILP Formulation," Computers \& Chemical Engineering, 17, 211-227 (1993).

The Outlook for the US Chemical Industry, KPMG, (2010) http://www.kpmg.de/docs/ 20101028US chemical industry outlook 2010.pd f)

Langevin, A., Mbaraga, P.; Campbell, J. F., "Continuous Approximation Models for Freight Distribution: An Overview," Transportation Research B, 30, 163-188 (1996).

Lee S. and Grossmann I.E., "New Algorithms for Nonlinear Generalized Disjunctive Programming," Computers and Chemical Engineering, 24, 2125-2141, (2000).

Li, Z. and M. Ierapetritou, "Production planning and scheduling integration through augmented Lagrangian optimization," Computers and Chemical Engineering 34, 996 - 1006 (2010).

Li, Z. and M. Ierapetritou, "Robust Optimization for Process Scheduling Under Uncertainty," Ind. Eng. Chem. Res., 47, 4148-4157 (2008).

Lima, R., I.E. Grossmann and Y. Jiao, "Long-term scheduling of a single-stage multi-product continuous process to manufacture high performance glass," Computers \& Chemical Engineering 35, 554-574 (2011).

Linderoth, J. and Wright, S. J., "Implementing a Decomposition Algorithm for Stochastic Programming on a Computational Grid," Computational Optimization and Applications, 24, 207-250 (2003).

Lindo Systems Inc., LINDOGlobal Solver (2010).

Liu, M. L. and N. V. Sahinidis, Optimization in process planning under uncertainty, Industrial \& Engineering Chemistry Research, 35(11), 4154-4165 (1996).

Liu, S., Pinto, J. M., \& L.G. Papageorgiou, "TSP-based MILP model for medium term planning of single-stage 
continuous multiproduct plants,". Industrial \& Engineering Chemistry Research, 47(20), 77337743 (2008).

Maravelias, C.T., Sung, C., "Integration of planning and scheduling: Overview, challenges, and opportunities," Computers and Chemical Engineering 33, 1919 - 1930 (2009).

Maravelias, C.T. and Grossmann, I. E., "A Hybrid MILP/CP Decomposition Approach for the Continuous Time Scheduling of Multipurpose Batch Plants," Computers and Chemical Engineering, 28, 19211949 (2004).

Mendez, C.A., J. Cerdá , I. E. Grossmann, I. Harjunkoski, and M. Fahl, "State-Of-The-Art Review of Optimization Methods for Short-Term Scheduling of Batch Processes," Computers \& Chemical Engineering 30, 913-946 (2006).

Mendez, C.A., I.E. Grossmann, I. Harjunkoski and P. Kabore, "A Simultaneous Optimization Approach for Offline Blending and Scheduling of Oil-refinery Operations," Computers and Chemical Engineering, 30, 614-634 (2006).

Milano, M. (Ed.), Constraint and Integer Programming Toward a Unified Methodology, Kluwer Academic Publishers (2003).

Mouret, S., I.E. Grossmann and P. Pestiaux, "A Novel Priority-Slot Based Continuous-Time Formulation for Crude-Oil Scheduling Problems," Ind.Eng.Chem. Res. 48, 8515-8528 (2009).

Neiro S.M.S. and J. M. Pinto, “ Lagrangean Decomposition Applied to Multiperiod Planning of Petroleum Refineries under Uncertainty," Latin American Applied Research, 36, 213 - 220 (2006).

Nystrom, R. H., Franke, R., Harjunkoski, I., Kroll, A., "Production Campaign Planning Including Grade Transition Sequencing and Dynamic Optimization," Comput. Chem. Eng. 29 (10), 2163-2179 (2005).

Pantelides, C.C. (1994). Unified Frameworks for Optimal Process Planning and Scheduling. Foundations of Computer-Aided Process Operations, CACHE publications, New York, 253-274.

Pekny, J.F., D.L. Miller and G.J. McRaec, “An Exact Parallel Algorithm for Scheduling when Production Costs Depend on Consecutive System States," Computers \& Chemical Engineering, 14, 1009-1023 (1990).

Perea, E., Ydstie, E. and Grossmann, I.E., "A Model Predictive Control Strategy for Supply Chain Optimization," Computers \& Chemical Engineering, 27, 1201-1218 (2003).

Pinedo, M. Scheduling: Theory, Algorithms, and Systems. Prentice Hall (2001).
Pinto, J. and I.E. Grossmann, "Optimal Cyclic Scheduling of Multistage Multiproduct Continuous Plants," Computers and Chemical Engineering, 18, 797-816 (1994)

Pistikopoulos, E., Georgiadis, M., Papageorgiou, L. Supply-chain Optimization, Vol.4. Wiley (2007).

Pistikopoulos, E., Georgiadis, M. and Dua, V., Multi-parametric programming: theory, algorithms, and applications, Vol. 1 Wiley (2007).

Pochet Y. and L. A. Wolsey, "Production Planning by Mixed Integer Programming," Springer, New York (2006).

Quesada I. and Grossmann I.E., "An LP/NLP Based Branch and Bound Algorithm for Convex MINLP Optimization Problems," Computers and Chemical Engineering, 16, 937-947 (1992).

Raman R. and Grossmann I.E., "Modelling and Computational Techniques for Logic-Based Integer Programming," Computers and Chemical Engineering, 18, 563 (1994).

Ruiz, J.P. and I.E. Grossmann, "A hierarchy of relaxations for nonlinear convex generalized disjunctive programming," accepted for publication in. European Journal of Operational Research (2011).

Ruszczynski, A., "Decomposition Methods in Stochastic Programming," Math. Programming (Ser. B) 79, 333-353 (1997).

Sahinidis, N., "BARON: A General Purpose Global Optimization Software Package," Journal of Global Optimization 8(2) 201-205 (1996).

Sahinidis, N.V., "Optimization under Uncertainty: State of the Art and Opportunities," Computers and Chemical Engineering 28, 971-983 (2004).

Sawaya, N. and I.E. Grossmann, "Reformulations, Relaxations and Cutting Planes for Linear Generalized Disjunctive Programming," to appear in European Journal of Operational Research (2011).

Schweiger C.A. and C.A. Floudas. "Process Synthesis, Design and Control: A Mixed Integer Optimal Control Framework", Proceedings of DYCOPS-5 on Dynamics and Control of Process Systems, pp. 189-194 (1998).

Shah, N., Pantelides, C. C., \& Sargent, W. H., "A General Algorithm for Short-term Scheduling of Batch Operations-II. Computational Issues," Computers and Chemical Engineering, 2, 229 - 244 (1993).

Shah, N., "Pharmaceutical Supply Chains: Key Issues and Strategies for Optimization," Computers and Chemical Engineering 28, 929-941 (2004).

Shah, N.K., Z. Li and M.G. Ierapetritou, "Petroleum Refining Operations: Key Issues, Advances and Opportunities," Industrial \& Engineering Chemistry Research, 50(30), 1161-1170 (2011). 
Shapiro, J.F., Modeling the Supply Chain, Duxbury, Pacific Grove (2001).

Soyster, A. L., "Convex Programming with Set-inclusive Constraints and Applications to Inexact Linear Programming," Operations Research 21, 1154 (1973).

Stubbs R. and Mehrotra,S., “A Branch-and-Cut Method for 0-1 Mixed Convex Programming," Math Programming, $86: 3,515-532$ (1999).

Sundaramoorthy, A.; Maravelias, C. T. "A general framework for process scheduling.” AIChE J., 57(3), 695-710, (2011)

Tarhan, B., V. Goel and I.E. Grossmann, "A Multistage Stochastic Programming Approach for the Planning of Offshore Oil or Gas Field Infrastructure under Decision Dependent Uncertainty," Ind. Eng. Chem. Research 48, 3078-3097 (2009).

Tarhan, B., I.E. Grossmann and V. Goel, "Computational Strategies for Non-convex Multistage MINLP Models with Decision-Dependent Uncertainty and Gradual Uncertainty Resolution," submitted for publication (2010).

Tawarmalani, M. and Sahinidis, N., "Convexification and Global Optimization in Continuous and MixedInteger Nonlinear Programming," Kluwer Academic Publishers, (2002).

Tayur, S., Ganeshan, R. and Magazine, M. (Eds.), "Quantitative Models for Supply Chain Management," Kluwer Academic, Norwell, Massachusetts (1999).

Terrazas-Moreno and I. E. Grossmann, "A multiscale decomposition method for the optimal planning and scheduling of multisite continuous multiproduct plants," Chemical Engineering Science 664307 4318 (2011).

Terrazas-Moreno, S., P. Trotter and I.E. Grossmann, "Temporal and spatial Lagrangean decompositions in multi-site, multi-period production planning problems with sequencedependent changeovers," Computers and Chemical Engineering 35, 2913- 2928 (2011).

Van Hentenryck, P., The OPL Optimization Programming Language, MIT Press (1999).

Van Slyke, R.M. and R. Wets, "L-Shaped Linear Programs with Applications to Optimal Control and Stochastic Programming SIAM J. Appl.. Math., 17, 638-663 (1969).

.Varma, V. A., Reklaitis, G. V., Blau, G. E. and Pekny, J. F., "Enterprise-wide Modeling \& Optimization-an Overview of Emerging Research Challenges and Opportunities", Computers \& Chemical Engineering 31, 692 (2007).
Vecchietti, A. and I.E. Grossmann, "LOGMIP: A Disjunctive 0-1 Nonlinear Optimizer for Process Systems Models, Computers and Chemical Engineering 23, 555-565 (1999).

Verderame, P.M., Floudas, C.A. "Integrated Operational Planning and Medium-Term Scheduling for Large-Scale Industrial Batch Plant". Industrial and Engineering Chemistry Research 47, 4845 - 4860 (2008).

Viswanathan \& Grossmann I.E., "A Combined Penalty Function and Outer-approximation Method for MINLP Optimization," Computers \& Chemical Engineering, 14, Issue 7, July 1990, Pages 769-782 (1990).

Wassick, J.M., Enterprise-wide optimization in an integrated chemical complex Computers \& Chemical Engineering, 33 pp. 1950-1963 (2009).

Westerlund, T. and Pettersson, F., "An Extended Cutting Plane Method for Solving Convex MINLP Problems," Computers and Chemical Engineering, vol. 19(Suppl.), pp. S131-S136 (1995).

Westerlund T. and Pörn R, Solving Pseudo-Convex Mixed Integer Optimization Problems by Cutting Plane Techniques. Optimization and Engineering, 3, 253-280 (2002).

Wolsey L.A., 1998, Integer programming. Wiley-Interscience, New York.

You, F., P.M. Castro and I.E. Grossmann, "Dinkelbach's Algorithm as an Efficient Method for Solving a Class of MINLP Models for Large-Scale Cyclic Scheduling Problems," Computers and Chemical Engineering, 33, 1879-1889 (2009).

You, F., J.M. Pinto, E. Capón, I.E. Grossmann, N. Arora and L. Megan, "Optimal Distribution-Inventory Planning of Industrial Gases: I. Fast Computational Strategies for Large-Scale Problems," Ind. Eng Chem. Res. 50, 29102927 (2011).

You, F. and I.E. Grossmann, "Mixed-Integer Nonlinear Programming Models and Algorithms for Large-Scale Supply Chain Design with Stochastic Inventory Management," I\&EC Research 47, $7802-7817$ (2008).

You, F. and I.E. Grossmann, "Design of Responsive Process Supply Chains under Demand Uncertainty," Computers \& Chemical Engineering, 32, 3090-3111 (2008).

You, F., J.M. Wassick and I.E. Grossmann, "Risk Management for a Global Supply Chain Planning under Uncertainty: Models and Algorithms," AIChE J. 55, 931-946 (2009). 